Supporting information

\title{
Sustainable synthesis of renewable terpenoid-based (meth)acrylates using the CHEM21 green metrics toolkit
}

\author{
Martijn A. Droesbeke, Filip E. Du Prez*
}

Polymer Chemistry Research group, Centre of Macromolecular Chemistry (CMaC), Department of Organic and Macromolecular Chemistry, Faculty of Sciences, Ghent University, Krijgslaan 281-S4bis, B-9000 Ghent, Belgium

\section{* E-Mail: Filip.DuPrez@Ugent.be}

Table of Contents

1. Characterization of the terpenoids (meth)acrylates......................................S3

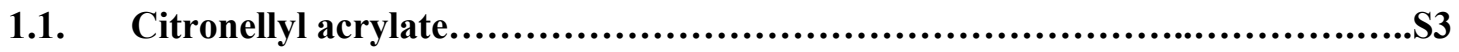

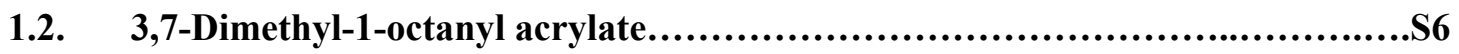

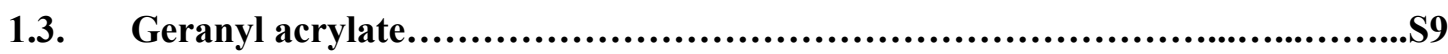

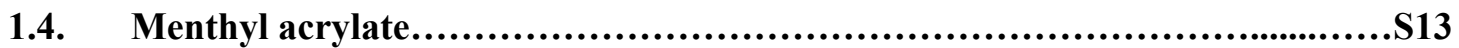

1.5. Citronellyl methacrylate...................................................

1.6. 3,7-Dimethyl-1-octanyl methacrylate..........................................S19

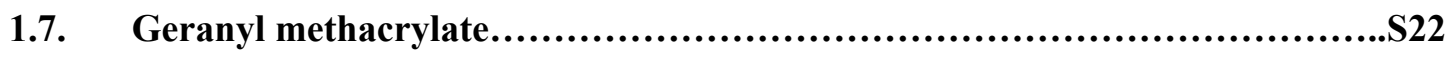

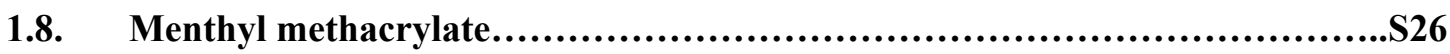

2. Detailed summary of the results obtained from the CHEM21 green metrics toolkit....S29

3. An example of the calculation of the Zero Pass for citronellyl acrylate using Method A...

4. An example of the calculation of the First Pass for citronellyl acrylate using Method A... 


\section{Characterization of the terpenoids (meth)acrylates}

\subsection{Citronellyl acrylate}

\subsubsection{NMR spectroscopy}

${ }^{1} \mathrm{H}$ NMR (400 MHz, chloroform- $d$ ) $\delta$ (ppm): 6.32 (dd, $\left.J=17.33,1.46 \mathrm{~Hz}, 1 \mathrm{H}\right), 6.01-6.09$ (m, $1 \mathrm{H}), 5.74$ (dd, $J=10.44,1.53 \mathrm{~Hz}, 1 \mathrm{H}), 5.02$ (tdt, $J=7.08,7.08,2.81,1.34,1.34 \mathrm{~Hz}, 1 \mathrm{H}), 4.07$ - 4.18 (m, 2 H), 1.84 - 1.99 (m, 2 H), 1.62 - 1.69 (m, 1 H), 1.61 (d, J=1.10 Hz, 3 H), 1.53 (s, 3 H), 1.47 - $1.52(\mathrm{~m}, 1 \mathrm{H}), 1.35$ - $1.47(\mathrm{~m}, 1 \mathrm{H}), 1.22$ - $1.35(\mathrm{~m}, 1 \mathrm{H}), 1.07$ - $1.22(\mathrm{~m}, 1 \mathrm{H}), 0.86$ $(\mathrm{d}, J=6.59 \mathrm{~Hz}, 3 \mathrm{H})$

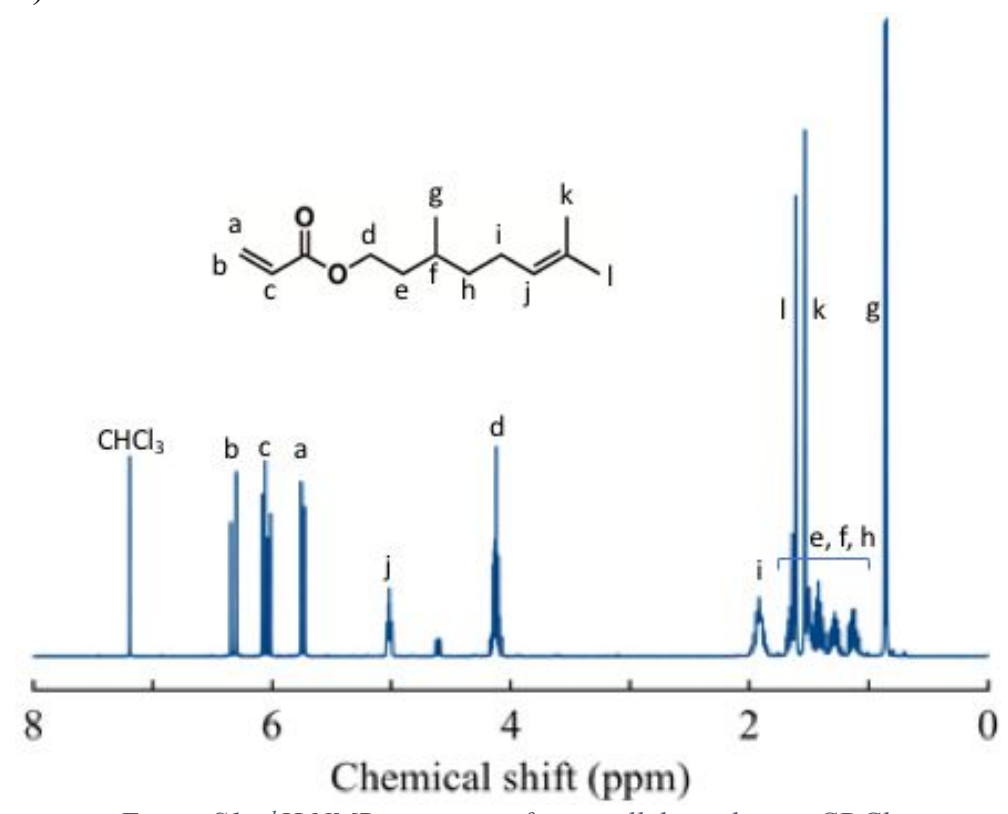

Figure $\mathrm{SI}-{ }^{1} \mathrm{H}$ NMR spectrum of citronellyl acrylate in $\mathrm{CDCl}_{3}$

${ }^{13} \mathrm{C}$ APT NMR (101 MHz, chloroform- $\left.d\right) \delta(\mathrm{ppm}): 166.34(\mathrm{C}), 130.41\left(\mathrm{CH}_{2}\right), 128.68(\mathrm{CH})$, $124.57(\mathrm{CH}), 109.78(\mathrm{C}), 63.11\left(\mathrm{CH}_{2}\right), 36.98\left(\mathrm{CH}_{2}\right), 35.42\left(\mathrm{CH}_{2}\right), 29.49(\mathrm{CH}), 25.72\left(\mathrm{CH}_{3}\right)$, $25.39\left(\mathrm{CH}_{2}\right), 19.44\left(\mathrm{CH}_{3}\right), 17.66\left(\mathrm{CH}_{3}\right)$

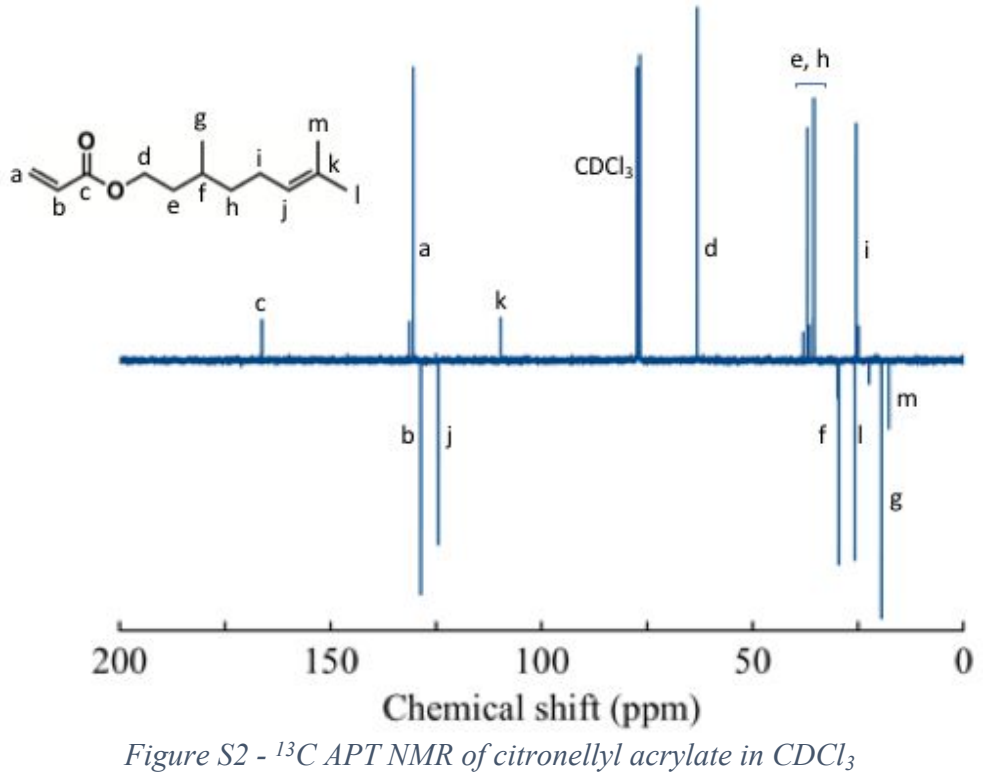




\subsubsection{ESI-MS spectrometry}

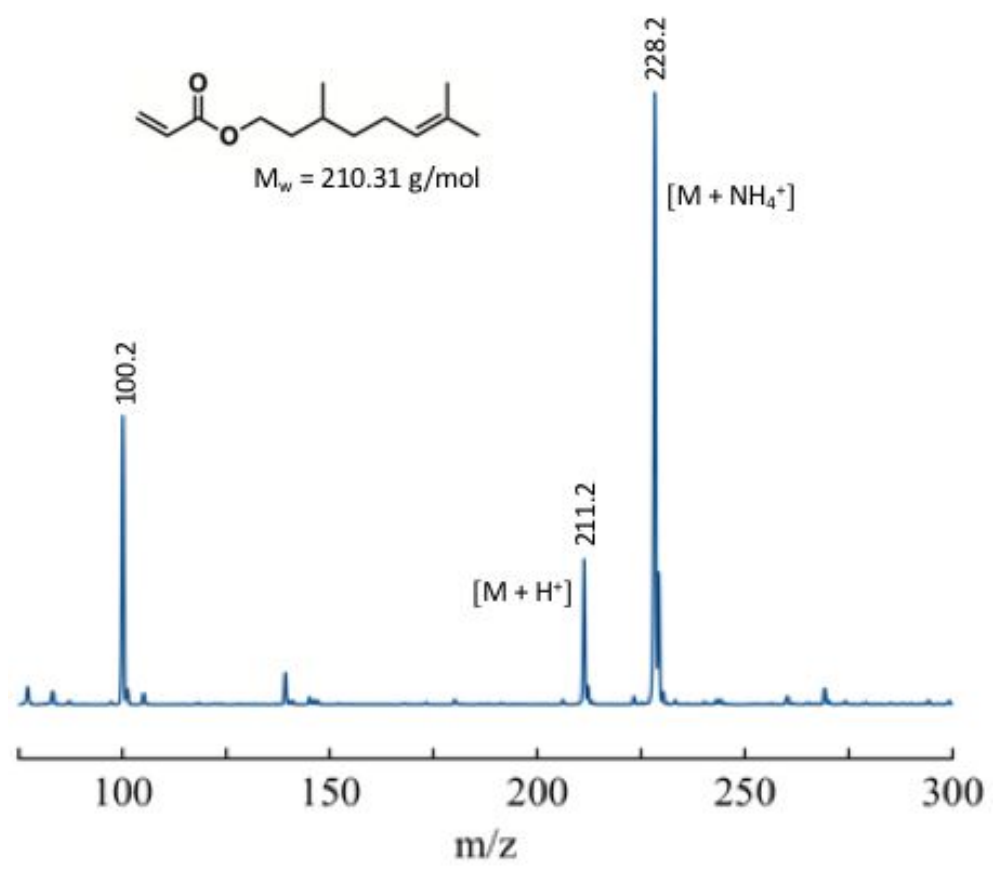

Figure S3 - ESI-MS spectrum of citronellyl acrylate

*Peak at 100.2 is a system artefact.

1.1.3. GC-CI-MS analysis
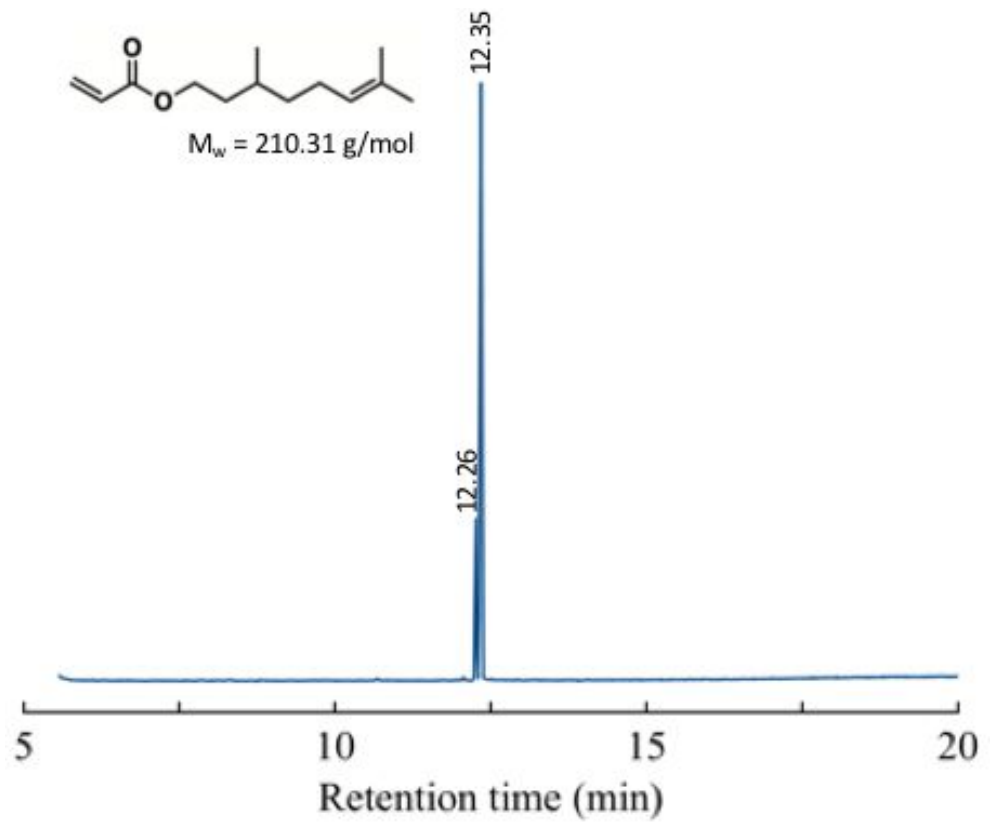

Figure S4 - GC spectrum obtained from the GC-CI-MS measurement of citronellyl acrylate

*The starting compound citronellol was a racemic mixture. 


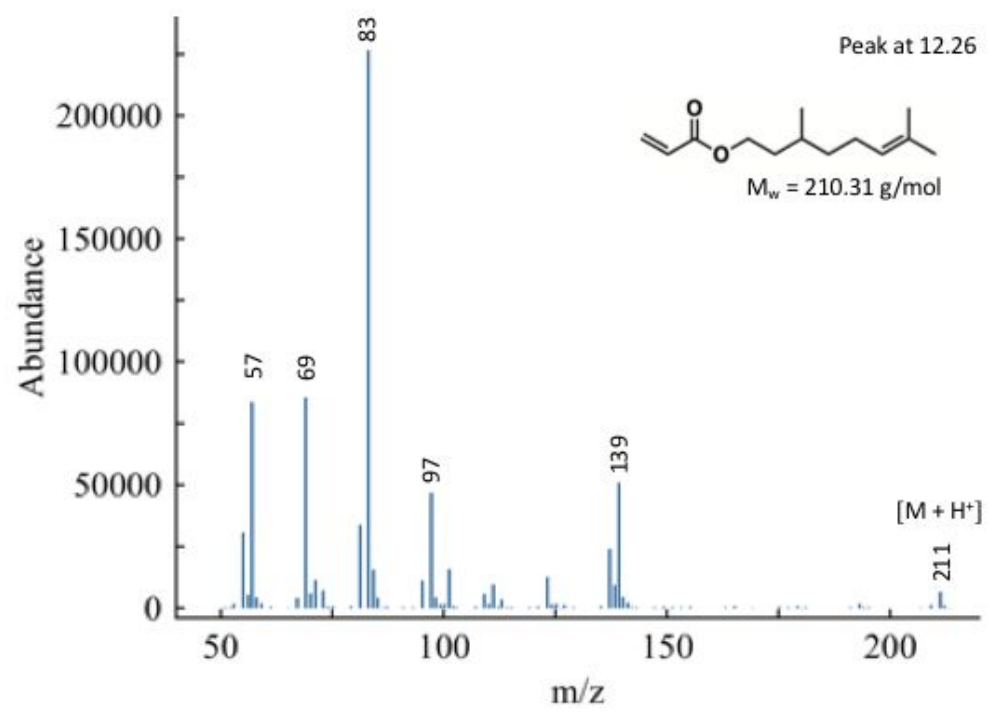

Figure S5 - CI-MS spectrum of citronellyl acrylate at a retention time of $12.26 \mathrm{~min}$

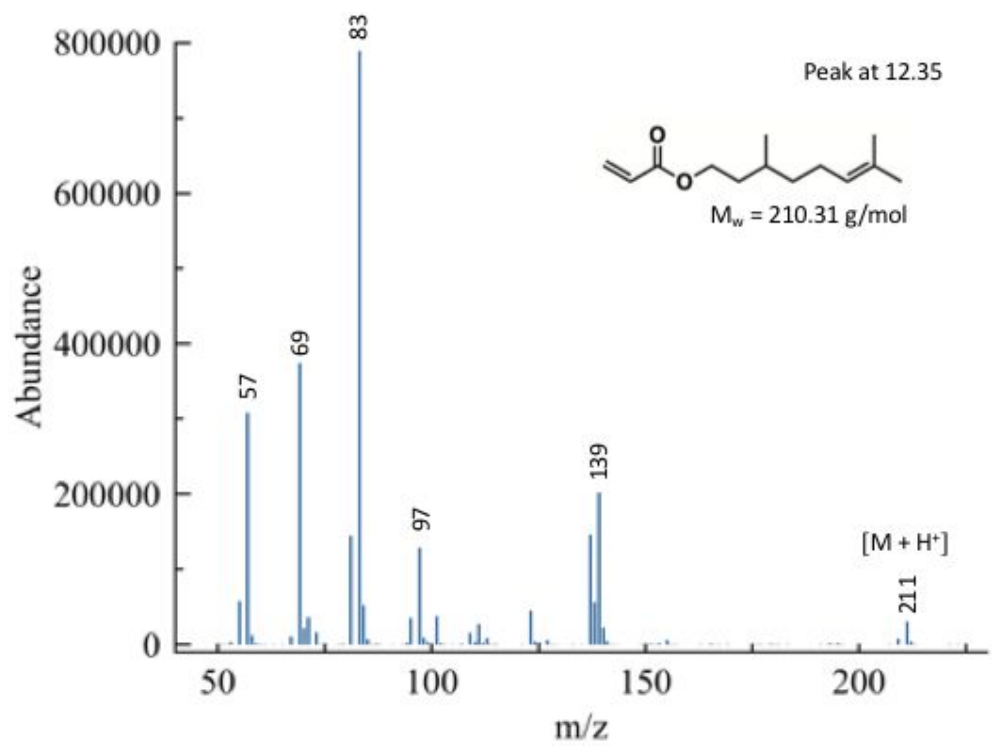

Figure S6 - CI-MS spectrum of citronellyl acrylate at a retention time of $12.35 \mathrm{~min}$

1.1.4. FTIR spectroscopy

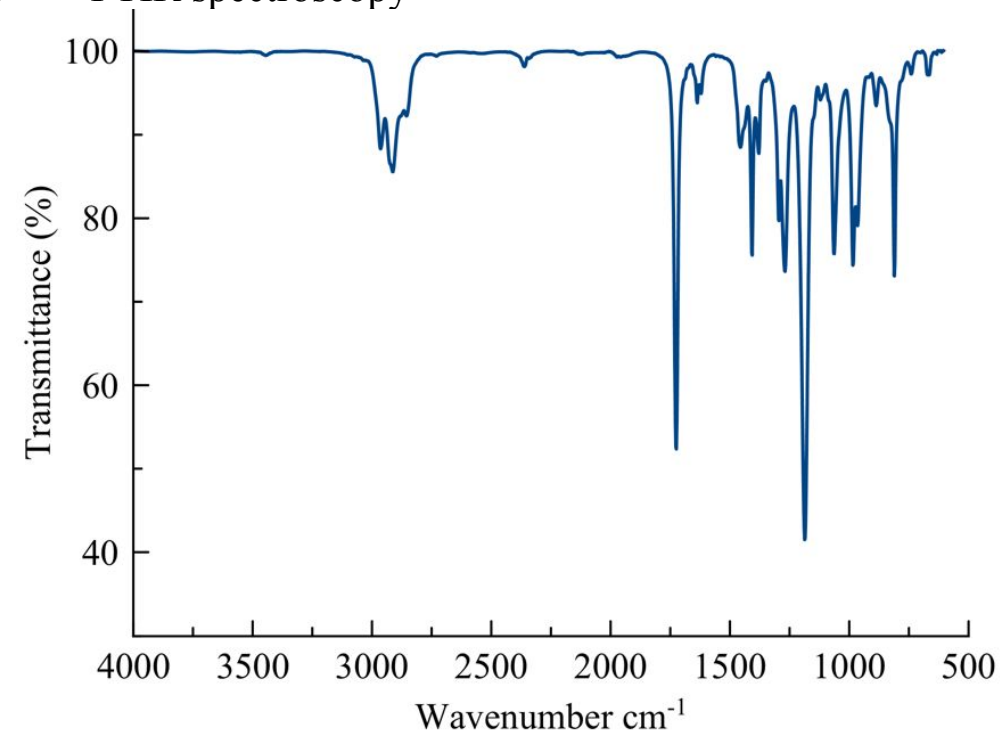

Figure S7 - FTIR spectrum of citronellyl acrylate 


\subsection{3,7-Dimethyl-1-octanyl acrylate}

\subsubsection{NMR spectroscopy}

${ }^{1} \mathrm{H}$ NMR (400 MHz, chloroform- $d$ ) $\delta(\mathrm{ppm}): 6.32(\mathrm{dd}, J=17.33,1.59 \mathrm{~Hz}, 1 \mathrm{H}), 6.01-6.09$ (m, $1 \mathrm{H}), 5.74(\mathrm{dd}, J=10.44,1.53 \mathrm{~Hz}, 1 \mathrm{H}), 4.07-4.17(\mathrm{~m}, 2 \mathrm{H}), 1.58-1.68(\mathrm{~m}, 1 \mathrm{H}), 1.36-1.55$ $(\mathrm{m}, 4 \mathrm{H}), 1.14-1.28(\mathrm{~m}, 3 \mathrm{H}), 1.03-1.14(\mathrm{~m}, 3 \mathrm{H}), 0.84(\mathrm{~d}, J=6.47 \mathrm{~Hz}, 3 \mathrm{H}), 0.80(\mathrm{~d}, J=6.59$ $\mathrm{Hz}, 6 \mathrm{H})$
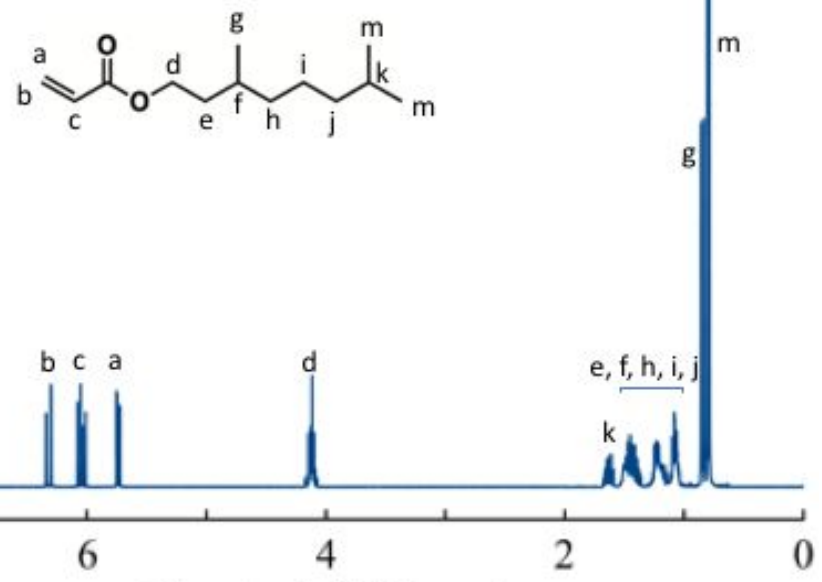

Chemical shift (ppm)

Figure S8 - ${ }^{1} \mathrm{H} N M R$ spectrum of 3,7-dimethyl-1-octanyl acrylate in $\mathrm{CDCl}_{3}$

${ }^{13} \mathrm{C}$ APT NMR (chloroform- $\left.d\right) \delta(\mathrm{ppm}): 166.36(\mathrm{C}), 130.40(\mathrm{C}), 128.69\left(\mathrm{CH}_{2}\right), 63.18\left(\mathrm{CH}_{2}\right)$, $39.20\left(\mathrm{CH}_{2}\right), 37.14\left(\mathrm{CH}_{2}\right), 35.50\left(\mathrm{CH}_{2}\right), 29.88(\mathrm{CH}), 27.96(\mathrm{CH}), 24.61\left(\mathrm{CH}_{2}\right), 22.69\left(\mathrm{CH}_{3}\right)$, $22.60\left(\mathrm{CH}_{3}\right), 19.55\left(\mathrm{CH}_{3}\right)$

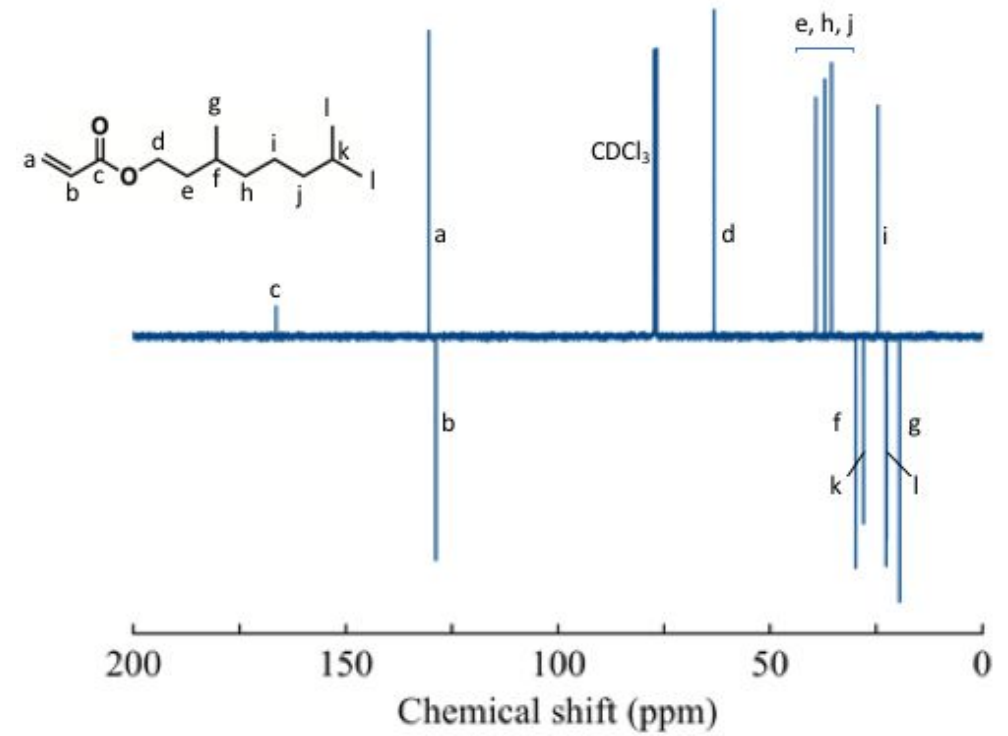

Figure $\mathrm{S} 9-{ }^{13} \mathrm{C}$ APT NMR spectrum of 3,7-dimethyl-1-octanyl acrylate in $\mathrm{CDCl}_{3}$ 


\subsubsection{ESI-MS spectrometry}

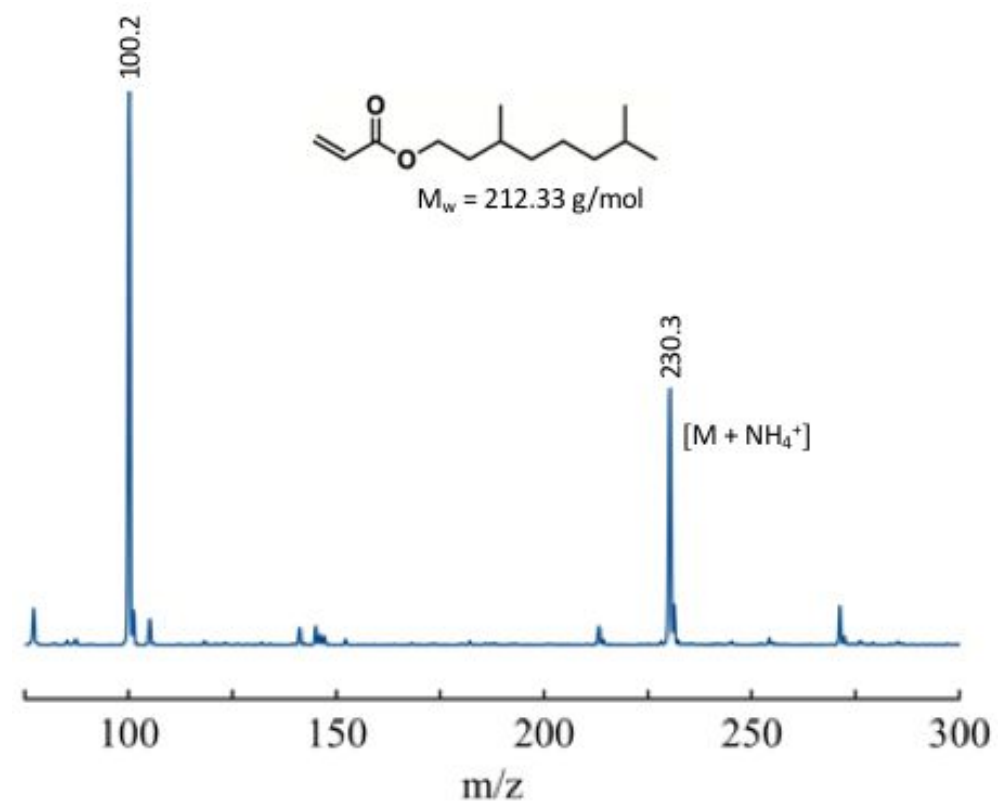

Figure S10 - ESI-MS spectrum of 3,7-dimethyl-1-octanyl acrylate

*Peak at 100.2 is a system artefact.

\subsubsection{GC-CI-MS analysis}

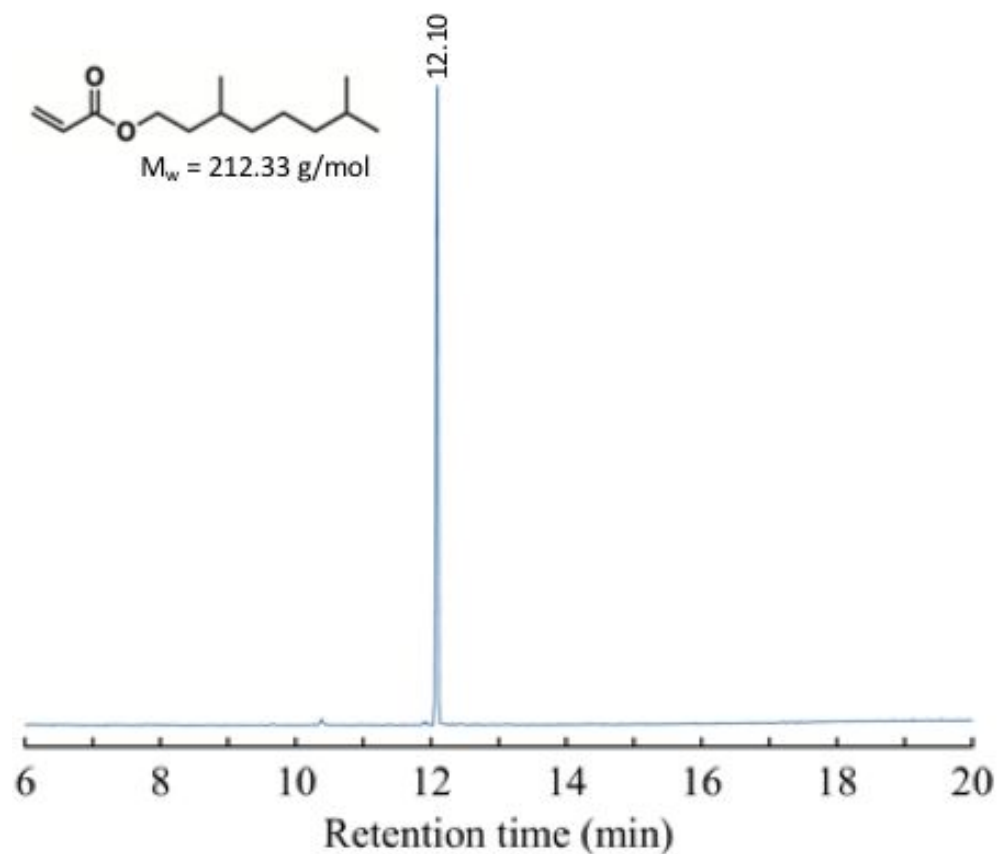

Figure S11 - GC spectrum obtained from the GC-CI-MS measurement of 3,7-dimethyl-1-octanyl acrylate 


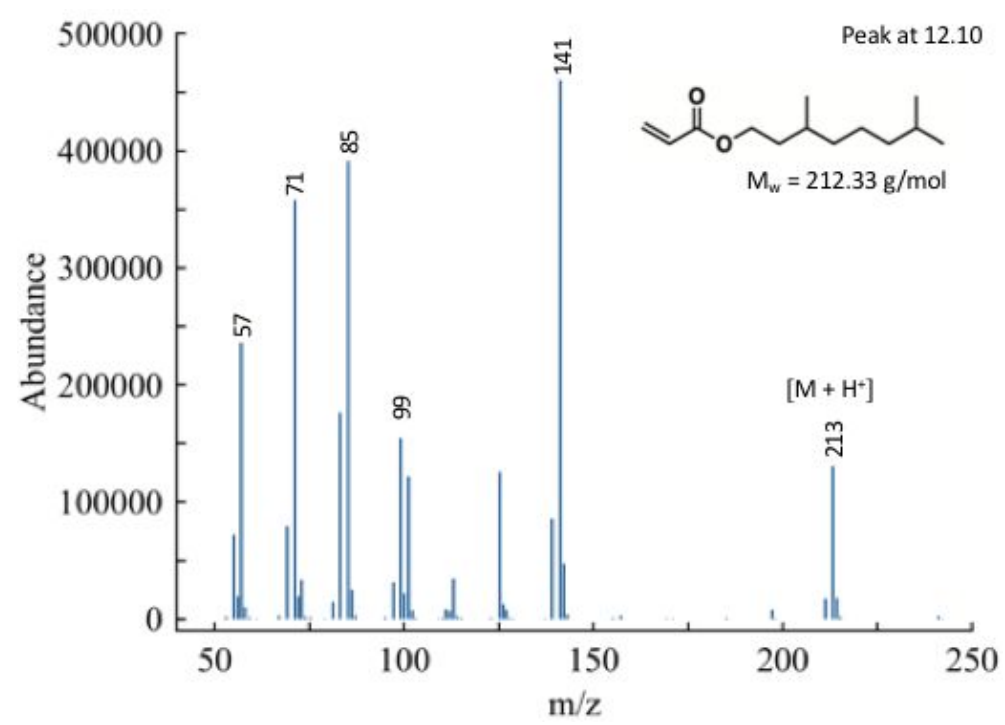

Figure S12 - CI-MS spectrum of 3,7-dimethyl-1-octanyl acrylate at $12.10 \mathrm{~min}$

\subsubsection{FTIR spectroscopy}

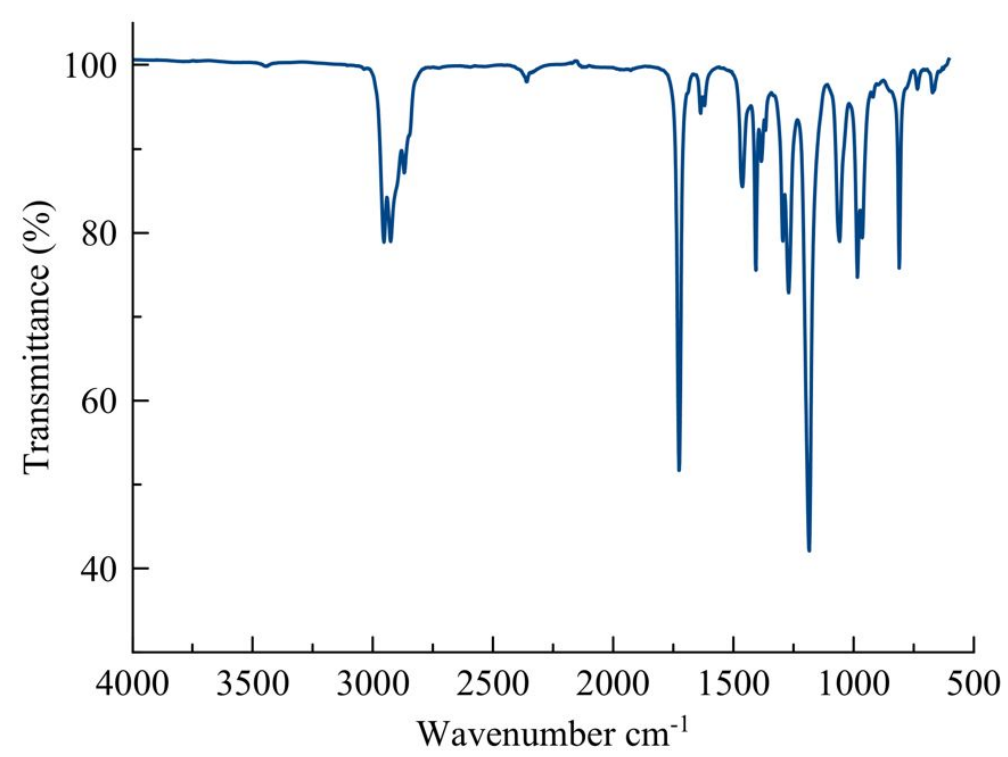

Figure S13 - FTIR spectrum of 3,7-dimethyl-1-octanyl acrylate 


\subsection{Geranyl acrylate}

\subsubsection{NMR spectroscopy}

${ }^{1} \mathrm{H}$ NMR (chloroform- $d$ ) $\delta$ (ppm): 6.34 (dd, $\left.J=17.33,1.46 \mathrm{~Hz}, 1 \mathrm{H}\right), 6.02-6.10(\mathrm{~m}, 1 \mathrm{H}), 5.74$ $(\mathrm{dd}, J=10.44,1.53 \mathrm{~Hz}, 1 \mathrm{H}), 5.31$ (tq, $J=7.10,1.25 \mathrm{~Hz}, 1 \mathrm{H}), 4.98-5.05(\mathrm{~m}, 1 \mathrm{H}), 4.61(\mathrm{~d}$, $J=7.08 \mathrm{~Hz}, 2 \mathrm{H}), 1.95-2.08(\mathrm{~m}, 4 \mathrm{H}), 1.65(\mathrm{~d}, J=0.61 \mathrm{~Hz}, 3 \mathrm{H}), 1.61(\mathrm{~d}, J=0.98 \mathrm{~Hz}, 3 \mathrm{H}), 1.53$ $(\mathrm{s}, 3 \mathrm{H})$

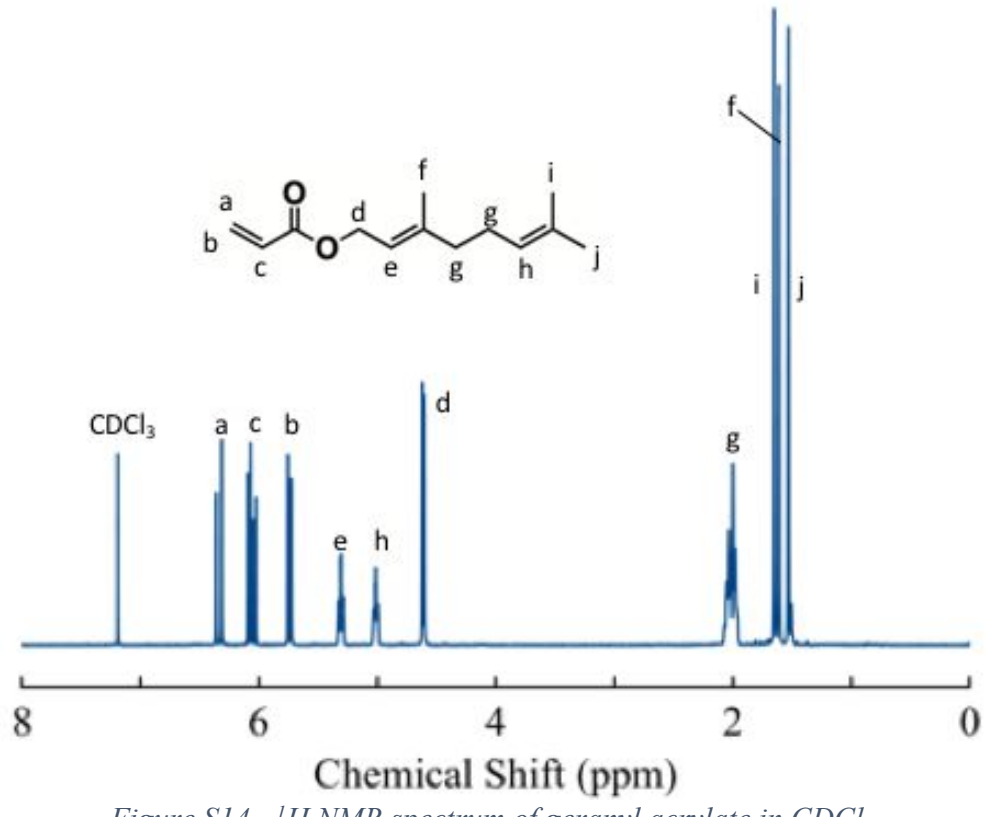

Figure $\mathrm{S} 14-{ }^{1} \mathrm{H} \mathrm{NMR}$ spectrum of geranyl acrylate in $\mathrm{CDCl}_{3}$

${ }^{13} \mathrm{C}$ APT NMR (chloroform- $\left.d\right) \delta(\mathrm{ppm}): 166.30(\mathrm{C}), 142.41(\mathrm{C}), 131.86(\mathrm{C}), 130.52\left(\mathrm{CH}_{2}\right)$, $128.63(\mathrm{CH}), 123.73(\mathrm{CH}), 118.20(\mathrm{CH}), 61.47\left(\mathrm{CH}_{2}\right), 39.54\left(\mathrm{CH}_{2}\right), 26.29\left(\mathrm{CH}_{2}\right), 25.68\left(\mathrm{CH}_{3}\right)$, $17.69\left(\mathrm{CH}_{3}\right), 16.50\left(\mathrm{CH}_{3}\right)$

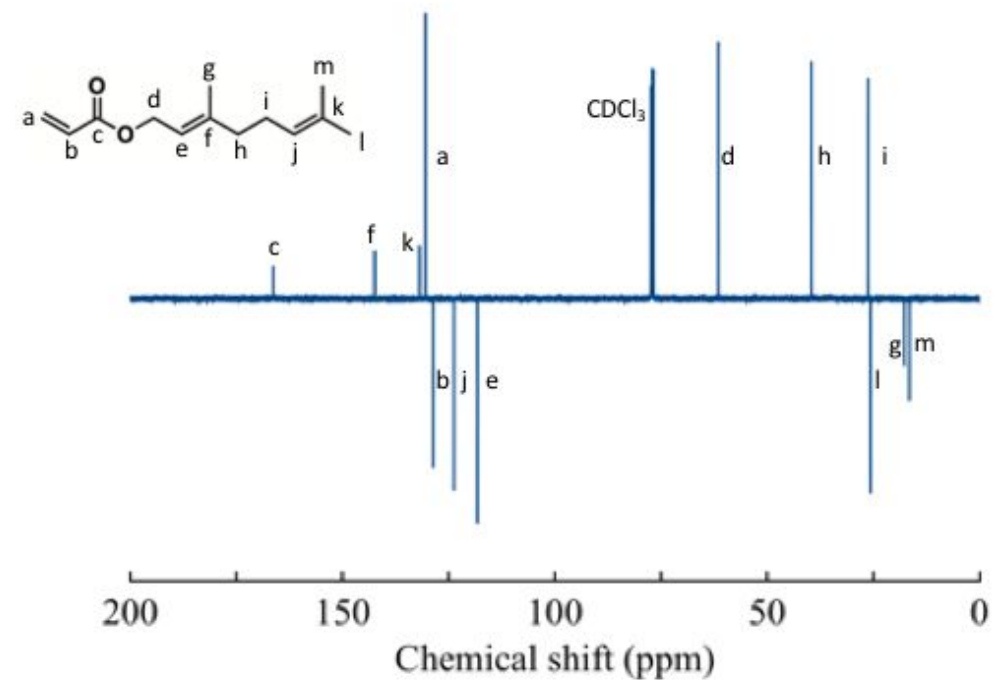

Figure $\mathrm{S} 15-{ }^{13} \mathrm{CAPT}$ NMR of geranyl acrylate in $\mathrm{CDCl}_{3}$ 


\subsubsection{ESI-MS spectrometry}

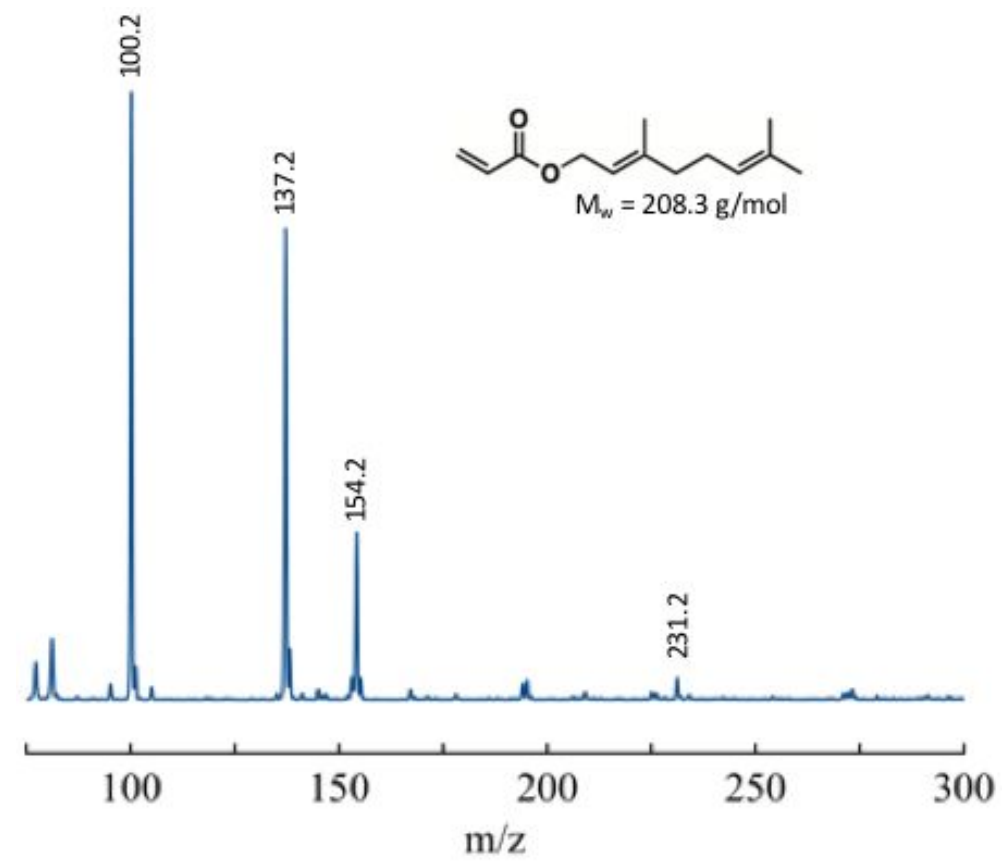

Figure S16 - ESI-MS of geranyl acrylate

There was a low visibility of the mass of geranyl acrylate at $231.2 \mathrm{~m} / \mathrm{z}$ because of fragmentation of the molecule during ESI-MS. The mass at $154.2 \mathrm{~m} / \mathrm{z}$ is geraniol and the peak at $137.2 \mathrm{~m} / \mathrm{z}$ corresponds to geraniol without the hydroxyl group. The peak at $100.2 \mathrm{~m} / \mathrm{z}$ is a system artefact.

\subsubsection{GC-CI-MS analysis}

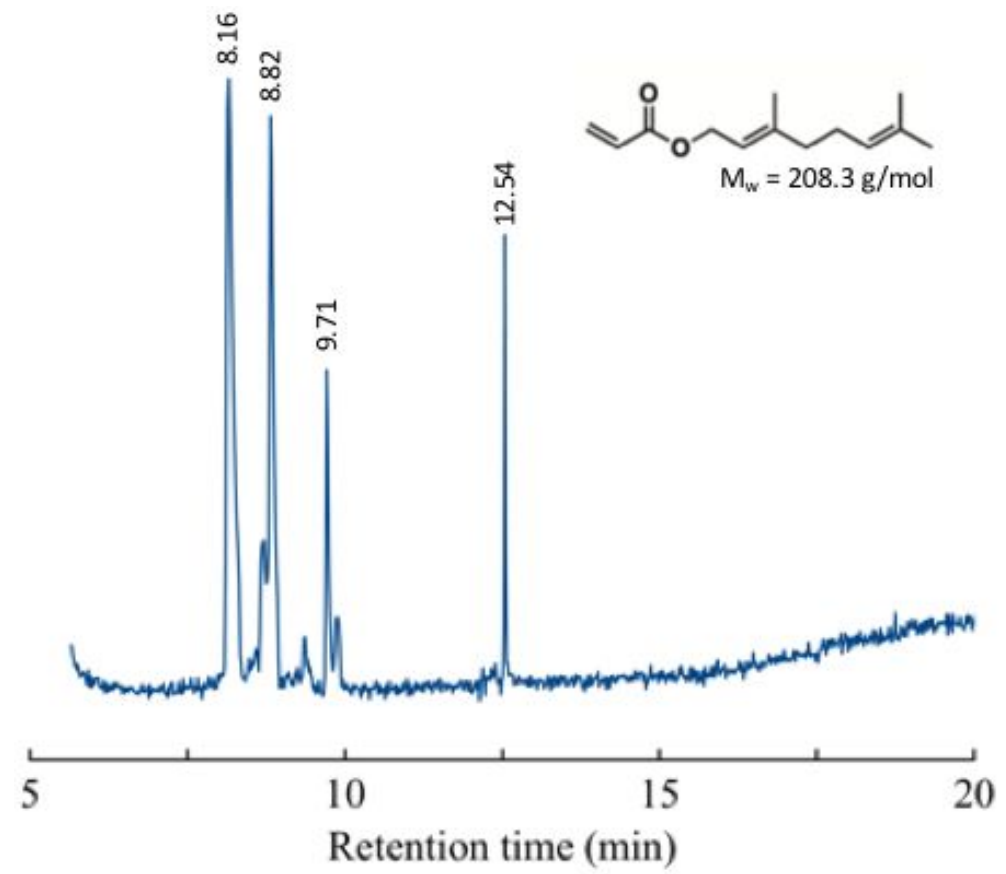

Figure S17 - GC spectrum obtained from the GC-CI-MS measurement of geranyl acrylate 


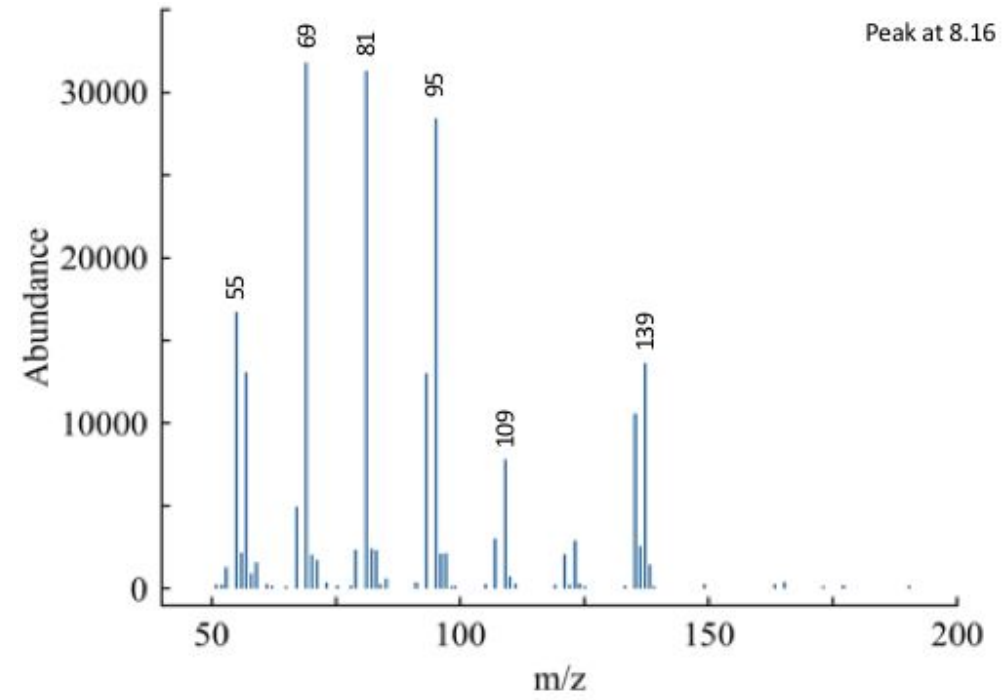

Figure S18 - CI-MS spectrum of a side product in the geranyl acrylate synthesis at $8.16 \mathrm{~min}$

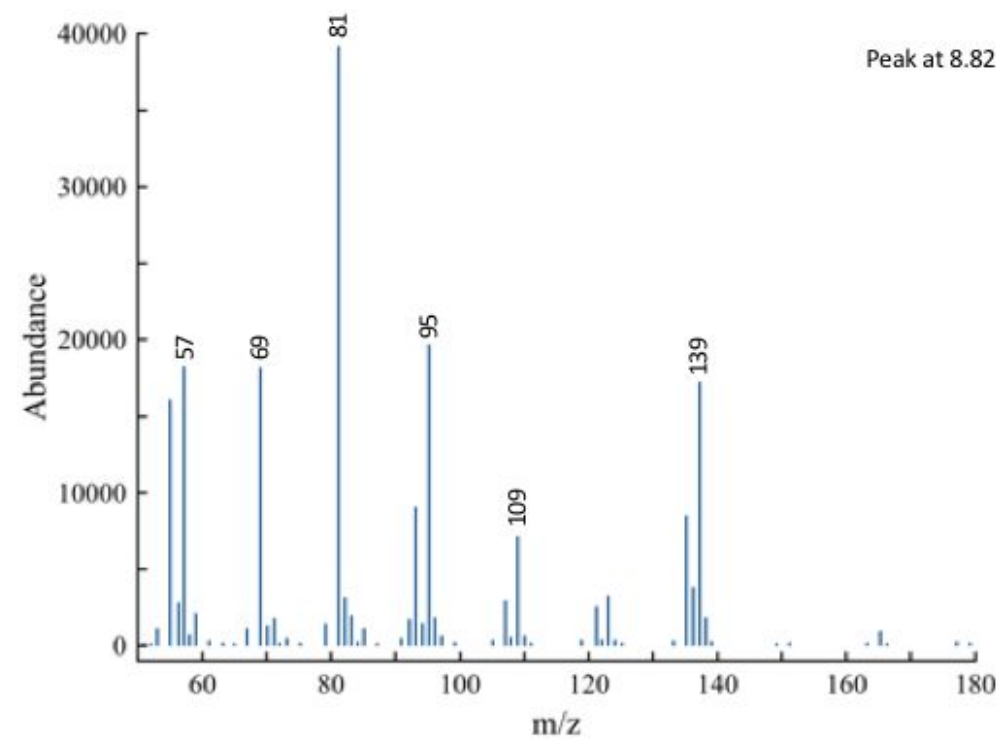

Figure S19-CI-MS spectrum of a side product in the geranyl acrylate synthesis at $8.82 \mathrm{~min}$

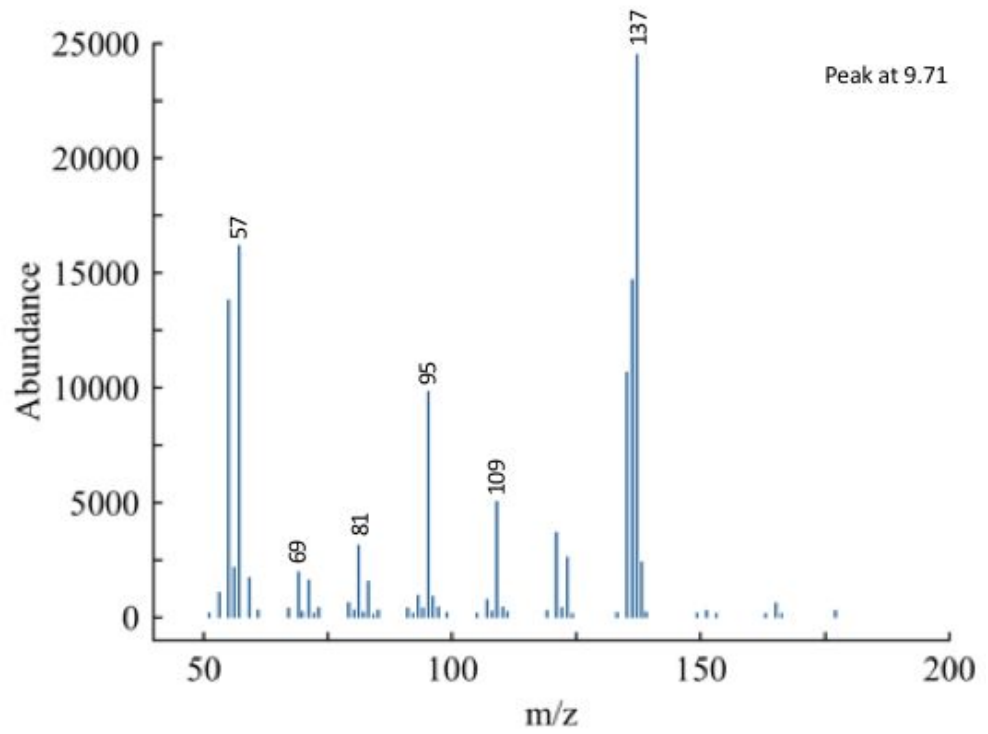

Figure S20 - CI-MS spectrum of a side product in the geranyl acrylate synthesis at $9.71 \mathrm{~min}$ 


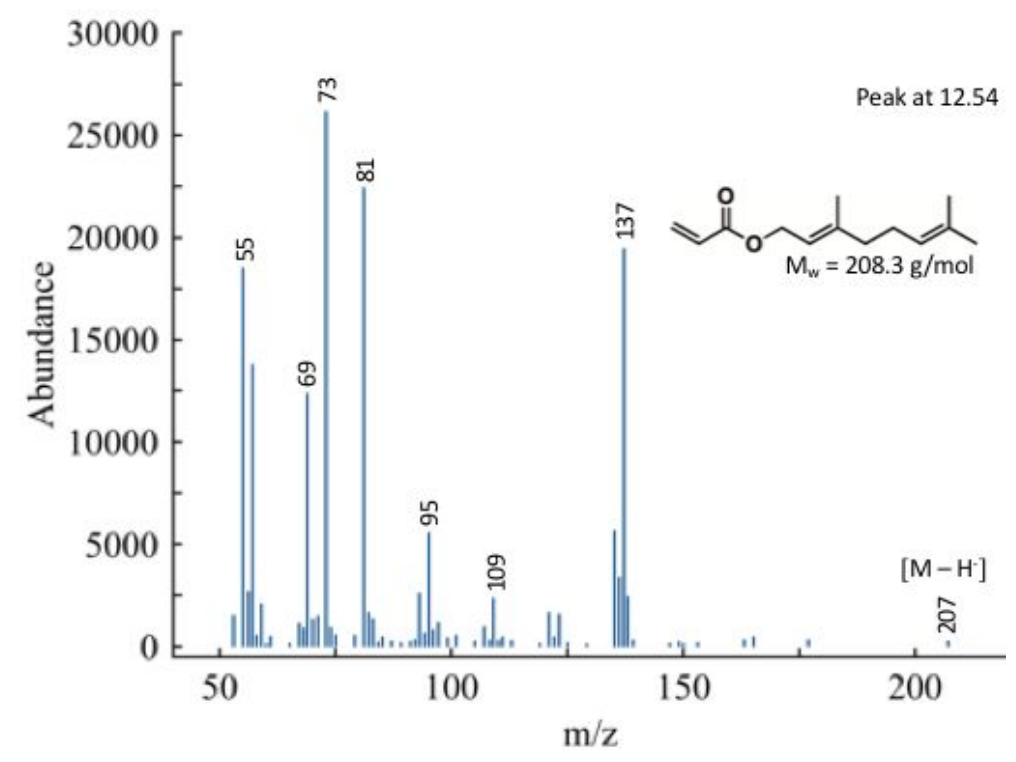

Figure S21 - CI-MS spectrum of geranyl acrylate synthesis at $12.54 \mathrm{~min}$

The degradation of geranyl acrylate already occurring during the heating step in GC-MS. The spectrums at $8.16,8.82$ and $9.71 \mathrm{~min}$ are coming from geraniol fragments from geranyl acrylate.

1.3.4. FTIR spectroscopy

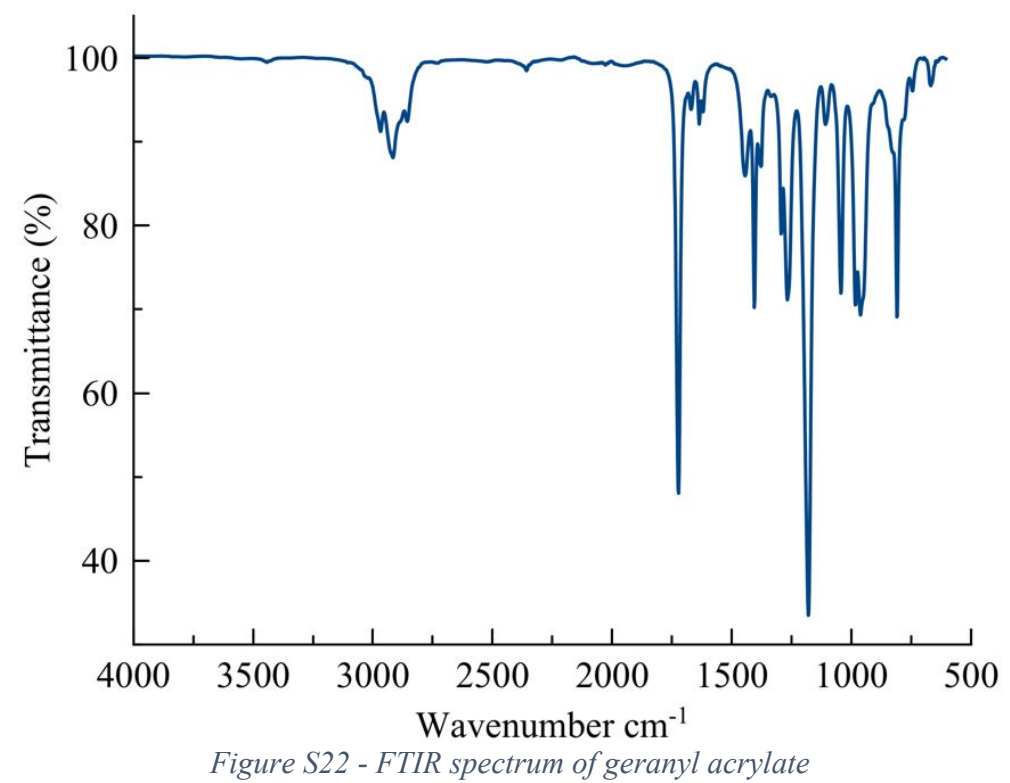

Figure S22 - FTIR spectrum of geranyl acrylate 


\subsection{Menthyl acrylate}

\subsubsection{NMR spectroscopy}

${ }^{1} \mathrm{H}$ NMR (chloroform- $d$ ) $\delta$ (ppm): 6.31 (dd, $J=17.33,1.59 \mathrm{~Hz}, 1 \mathrm{H}$ ), 6.04 (dd, $J=17.33,10.38$ Hz, $1 \mathrm{H}), 5.73$ (dd, $J=10.38,1.59 \mathrm{~Hz}, 1 \mathrm{H}), 4.69$ (td, $J=10.86,4.39 \mathrm{~Hz}, 1 \mathrm{H}), 1.93-1.99$ (m, 1 H), $1.80(\mathrm{dtd}, J=13.96,7.03,7.03,2.75 \mathrm{~Hz}, 1 \mathrm{H}), 1.58-1.66(\mathrm{~m}, 2 \mathrm{H}), 1.31-1.52(\mathrm{~m}, 2 \mathrm{H})$, $0.92-1.07$ (m, $2 \mathrm{H}), 0.83(\mathrm{dd}, J=6.77,5.80 \mathrm{~Hz}, 7 \mathrm{H}), 0.70(\mathrm{~d}, J=6.96 \mathrm{~Hz}, 3 \mathrm{H})$
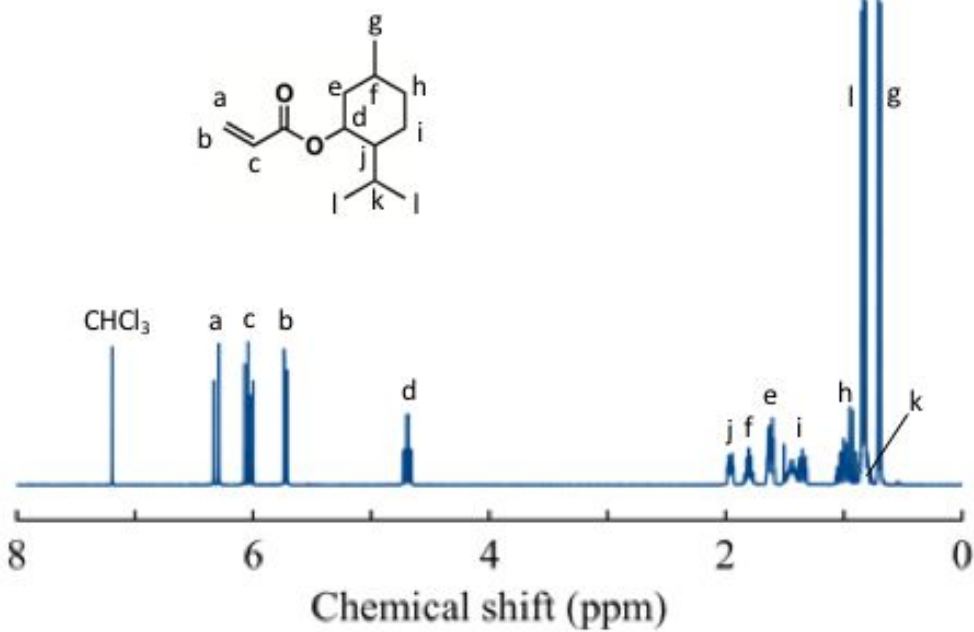

Figure $S 23-{ }^{1} \mathrm{H}$ NMR spectrum of menthyl acrylate in $\mathrm{CDCl}_{3}$

${ }^{13} \mathrm{C}$ APT NMR (chloroform- $\left.d\right) \delta(\mathrm{ppm}): 165.86(\mathrm{C}), 130.19\left(\mathrm{CH}_{2}\right), 129.06(\mathrm{CH}), 74.35\left(\mathrm{CH}_{2}\right)$, $47.11(\mathrm{CH}), 40.89\left(\mathrm{CH}_{2}\right), 34.29\left(\mathrm{CH}_{2}\right), 31.40(\mathrm{CH}), 26.35(\mathrm{CH}), 23.57\left(\mathrm{CH}_{2}\right), 22.03\left(\mathrm{CH}_{3}\right)$, $20.72\left(\mathrm{CH}_{3}\right), 16.44\left(\mathrm{CH}_{3}\right)$

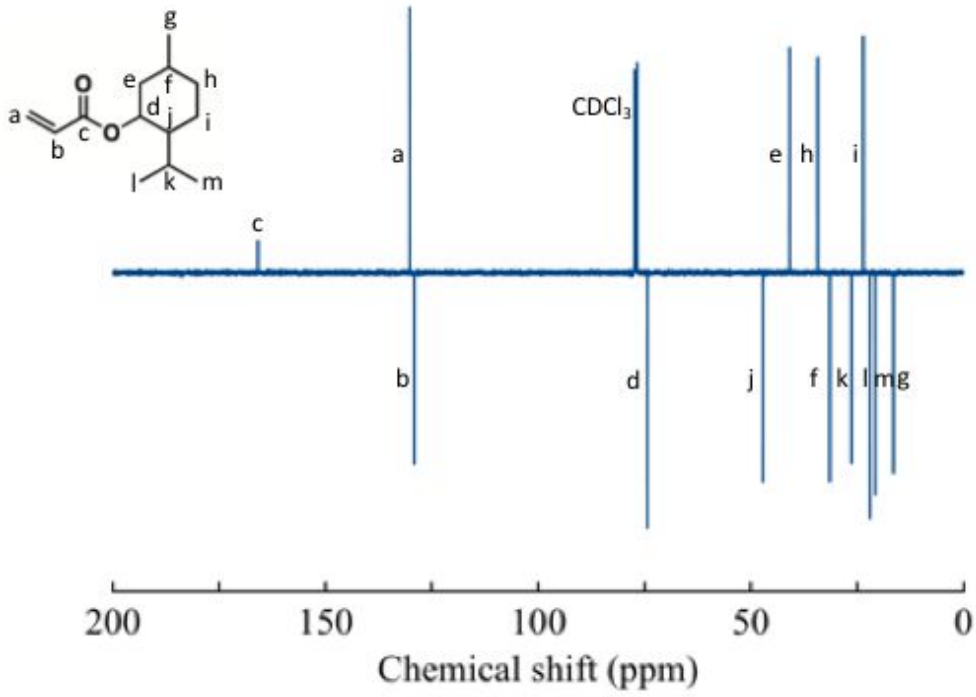

Figure $S 24-{ }^{13} \mathrm{C}$ APT NMR spectrum of menthyl acrylate in $\mathrm{CDCl}_{3}$ 


\subsubsection{ESI-MS spectrometry}

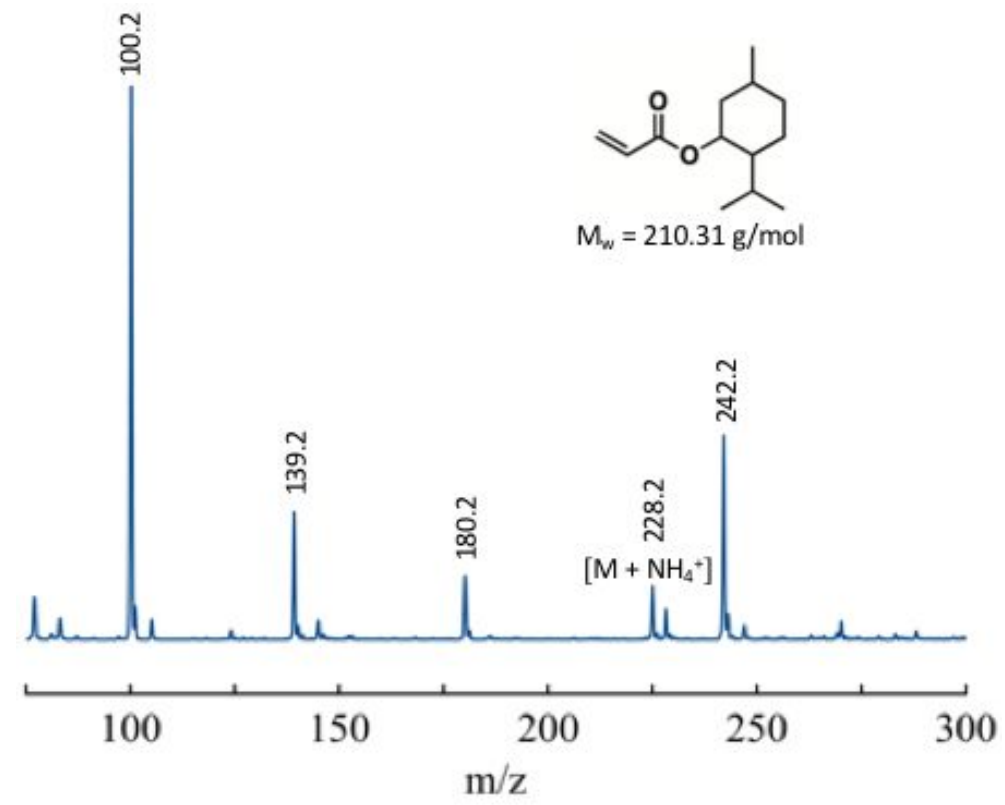

Figure S25 - ESI-MS spectrum of menthyl acrylate

Menthyl acrylate $\left[\mathrm{M}+\mathrm{NH}_{4}{ }^{+}\right]$was visible in the ESI-MS spectrum but so are other fragments of the compound. The peak at 100.2 is a system artefact.

\subsubsection{GC-CI-MS analysis}

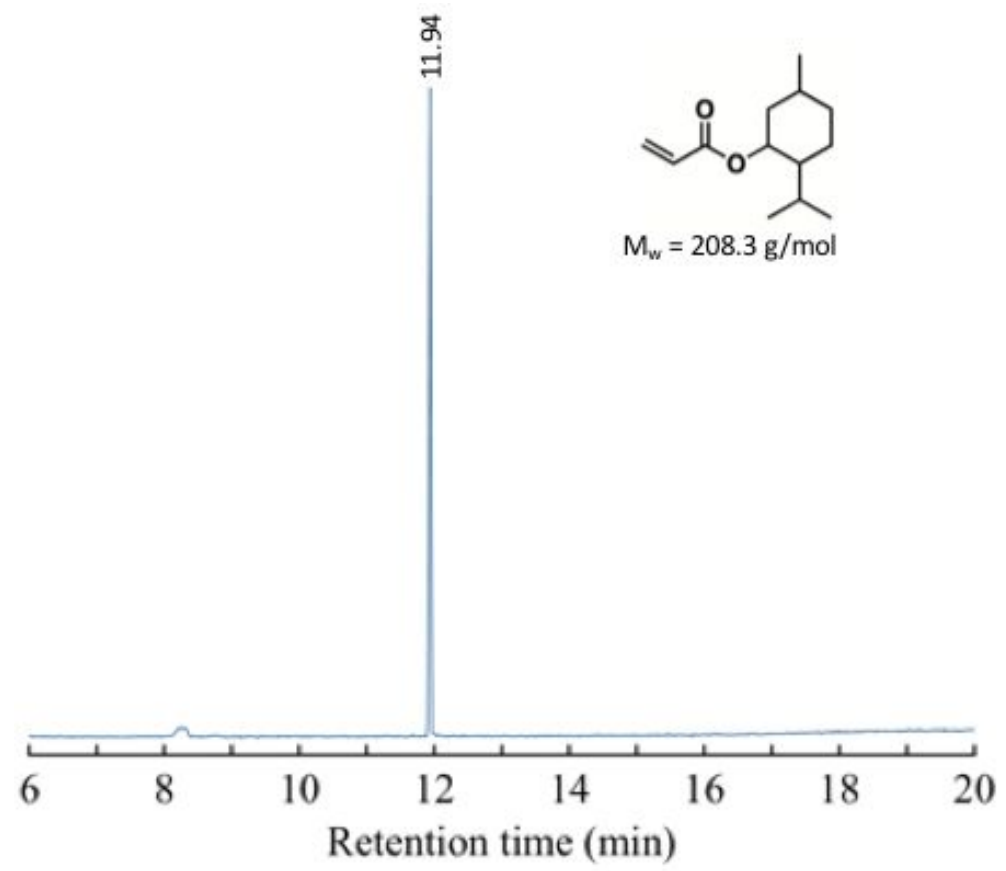

Figure S26 - GC spectrum obtained from the GC-CI-MS measurement of menthyl acrylate 


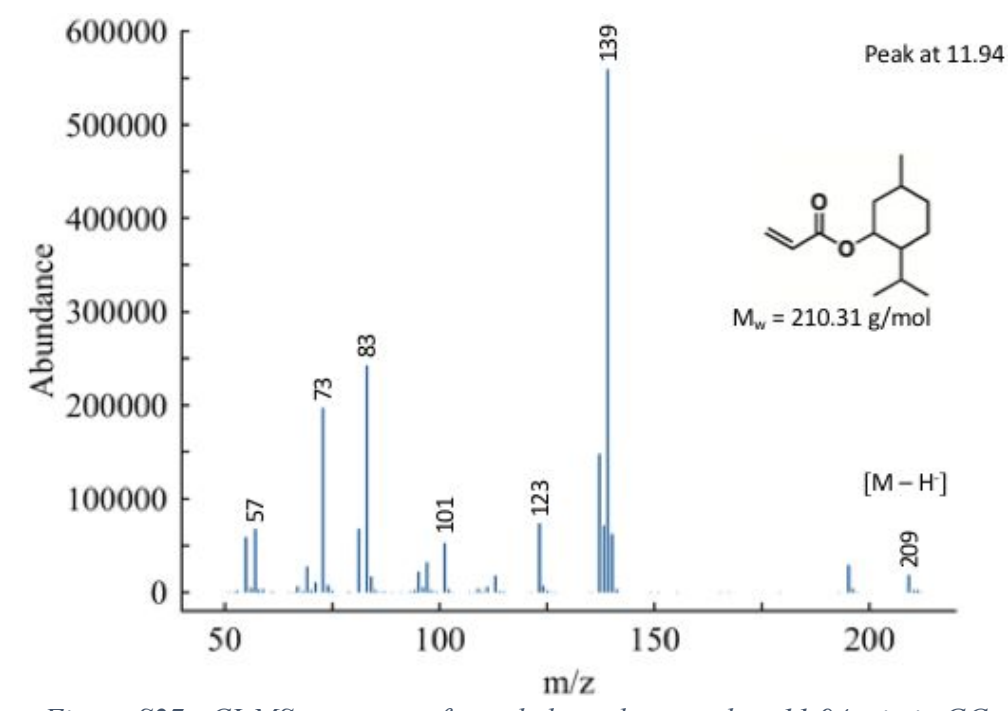

Figure S27 - CI-MS spectrum of menthyl acrylate, peak at 11.94 min in GC

\subsubsection{FTIR spectroscopy}

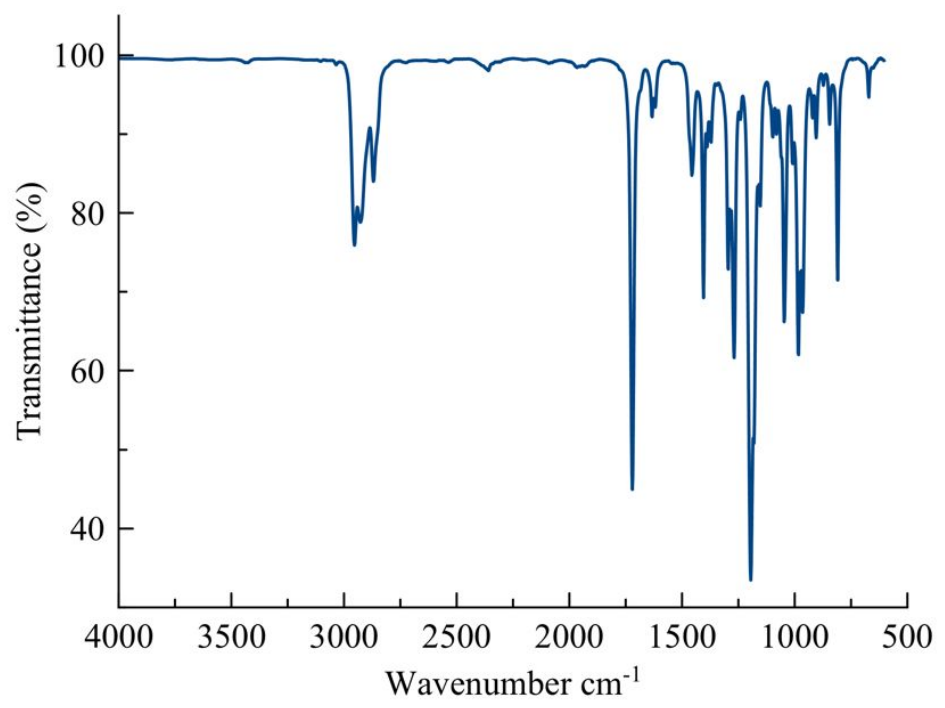

Figure S28 - FTIR spectrum of menthyl acrylate 


\subsection{Citronellyl methacrylate}

\subsubsection{NMR spectroscopy}

${ }^{1} \mathrm{H}$ NMR (chloroform- $d$ ) $\delta$ (ppm): $6.02(\mathrm{dd}, J=1.71,0.98 \mathrm{~Hz}, 1 \mathrm{H}), 5.47$ (quin, $J=1.62 \mathrm{~Hz}, 1 \mathrm{H}$ ), 4.99 - $5.05(\mathrm{~m}, 1 \mathrm{H}), 4.06$ - $4.16(\mathrm{~m}, 2 \mathrm{H}), 1.88-1.98(\mathrm{~m}, 2 \mathrm{H}), 1.84-1.88(\mathrm{~m}, 3 \mathrm{H}), 1.62$ - 1.69 $(\mathrm{m}, 1 \mathrm{H}), 1.61(\mathrm{~d}, J=1.10 \mathrm{~Hz}, 3 \mathrm{H}), 1.53(\mathrm{~s}, 3 \mathrm{H}), 1.37-1.51(\mathrm{~m}, 2 \mathrm{H}), 1.22-1.37(\mathrm{~m}, 1 \mathrm{H})$, $1.02-1.22(\mathrm{~m}, 1 \mathrm{H}), 0.86(\mathrm{~d}, J=6.59 \mathrm{~Hz}, 3 \mathrm{H})$
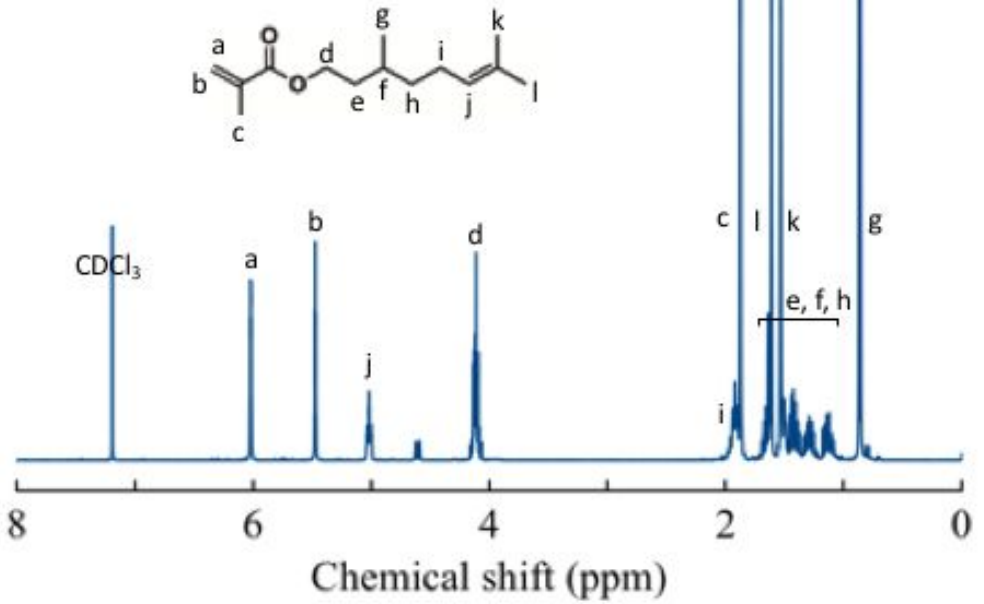

Figure $S 29-{ }^{1} \mathrm{H}$ NMR spectrum of citronellyl methacrylate in $\mathrm{CDCl}_{3}$

${ }^{13} \mathrm{C}$ APT NMR (chloroform- $d$ ) $\delta(\mathrm{ppm}): 167.57(\mathrm{C}), 136.57(\mathrm{C}), 131.37(\mathrm{C}), 125.14\left(\mathrm{CH}_{2}\right)$, $124.57(\mathrm{CH}), 63.22\left(\mathrm{CH}_{2}\right), 36.97\left(\mathrm{CH}_{2}\right), 35.42\left(\mathrm{CH}_{2}\right), 29.54(\mathrm{CH}), 25.71\left(\mathrm{CH}_{3}\right), 25.40\left(\mathrm{CH}_{2}\right)$, $19.48\left(\mathrm{CH}_{3}\right), 18.33\left(\mathrm{CH}_{3}\right), 17.66\left(\mathrm{CH}_{3}\right)$

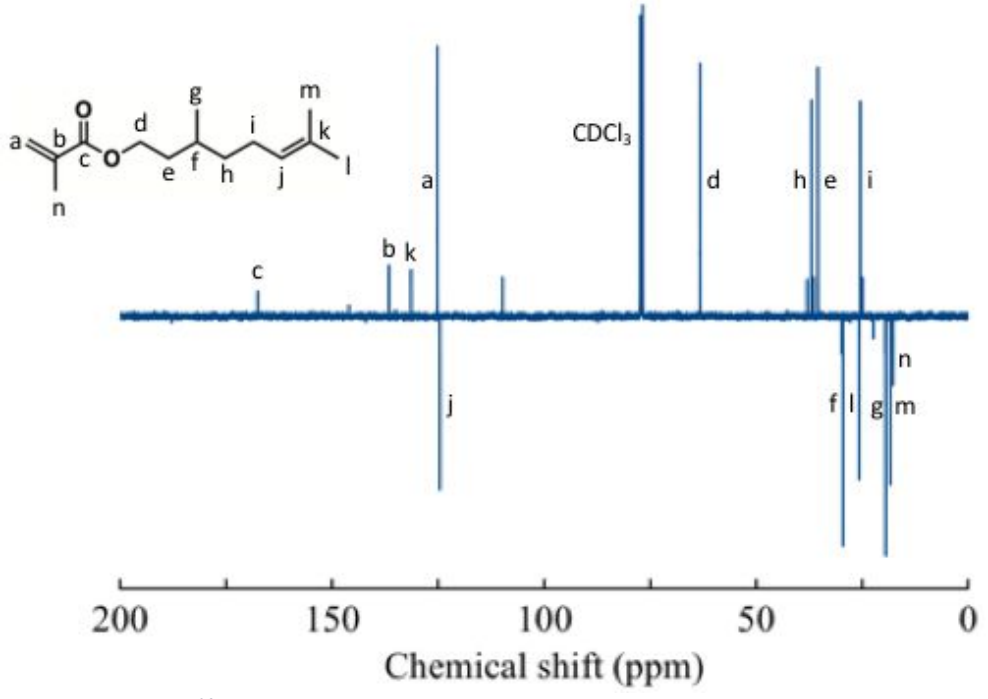

Figure $\mathrm{S} 30-{ }^{13} \mathrm{C}$ APT NMR spectrum of citronellyl methacrylate in $\mathrm{CDCl}_{3}$ 


\subsubsection{ESI-MS spectrometry}

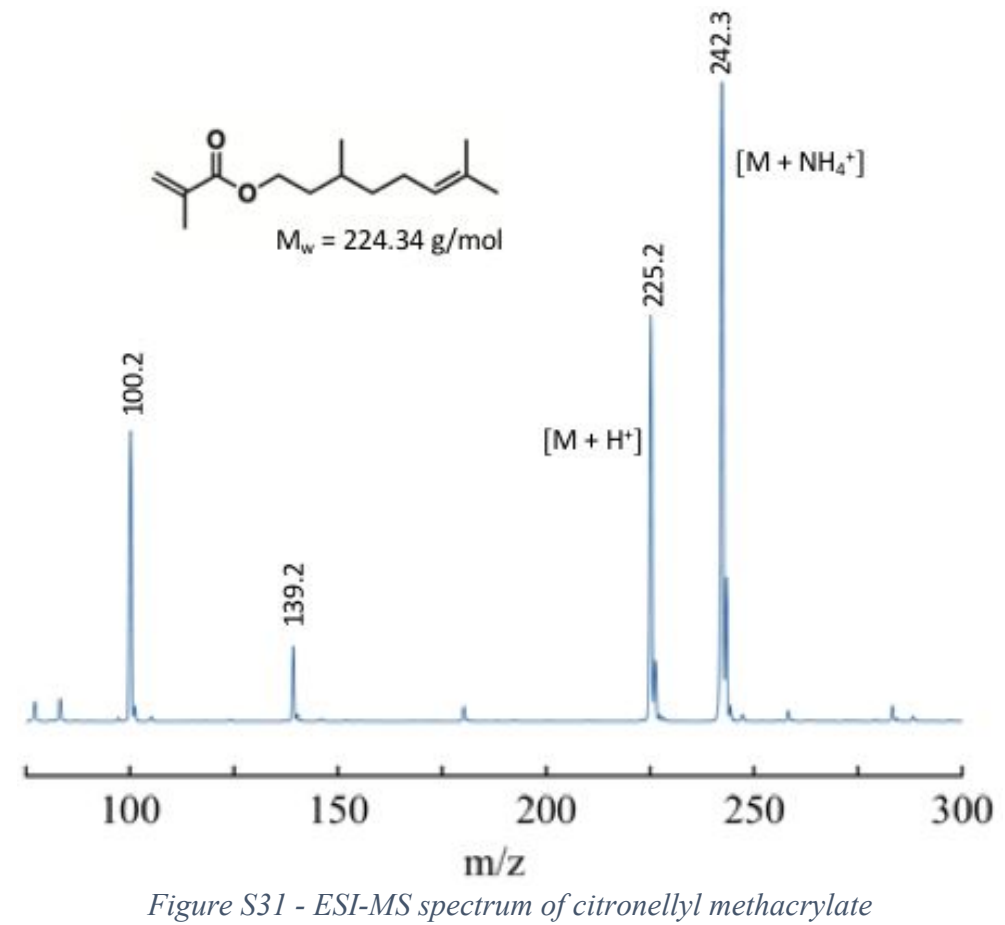

*Peak at 100.2 is a system artefact.

1.5.3. GC-CI-MS analysis

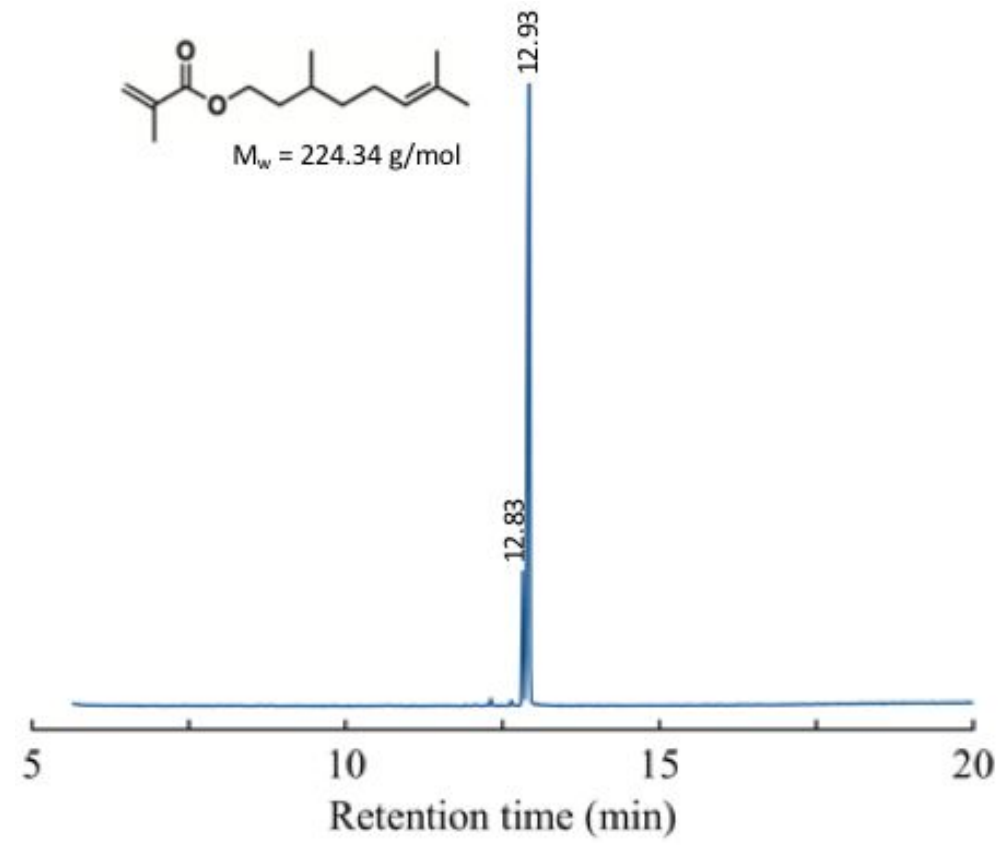

Figure S32 - GC spectrum obtained from the GC-CI-MS measurement of citronellyl methacrylate 


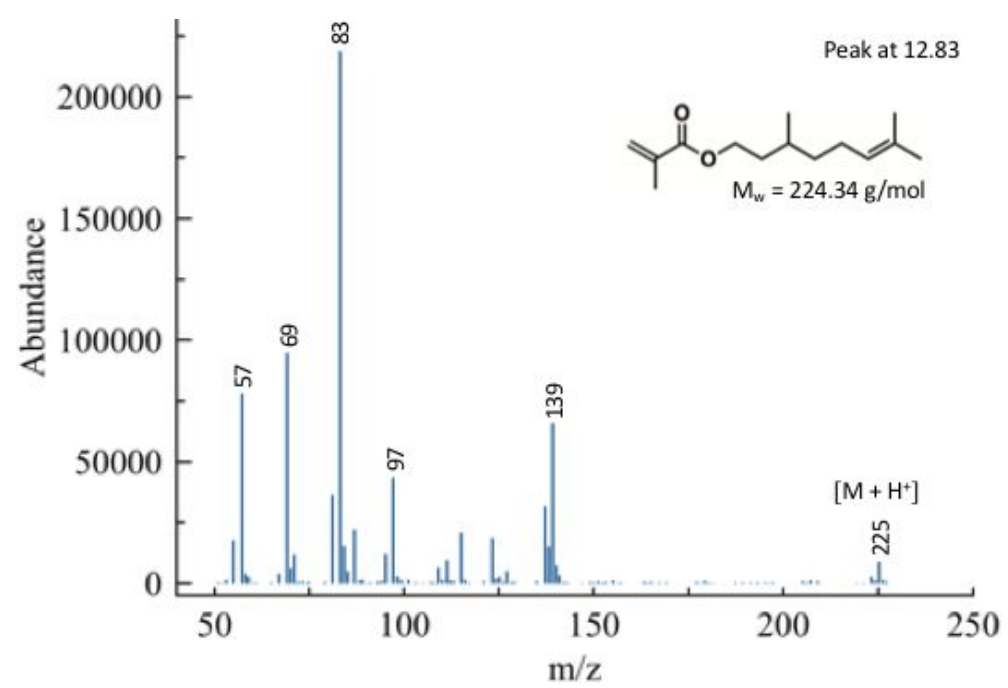

Figure S33 - CI-MS spectrum of citronellyl methacrylate, peak at $12.83 \mathrm{~min}$

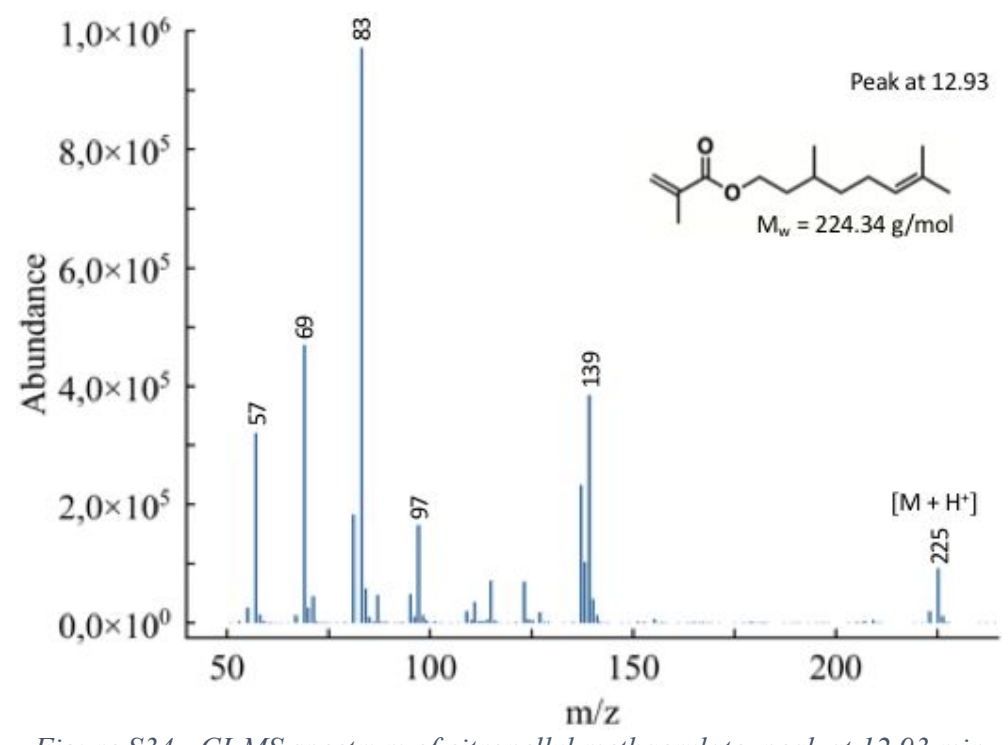

Figure S34 - CI-MS spectrum of citronellyl methacrylate, peak at $12.93 \mathrm{~min}$

\subsubsection{FTIR spectroscopy}

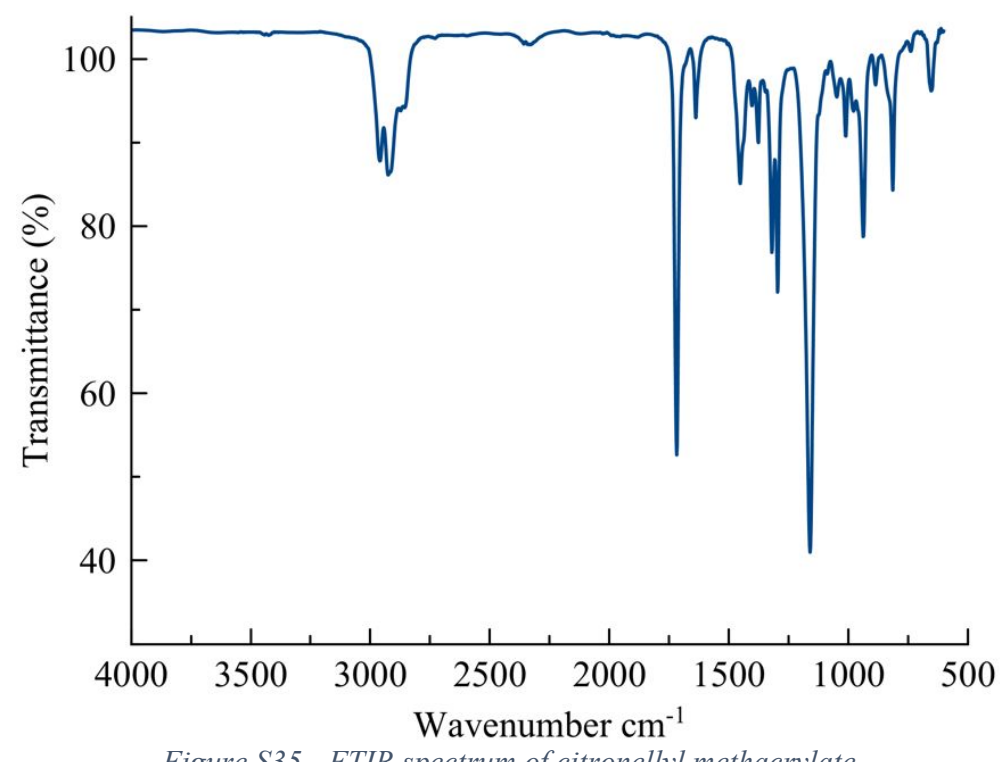

Figure S35 - FTIR spectrum of citronellyl methacrylate 


\subsection{3,7-Dimethyl-1-octanyl methacrylate}

\subsubsection{NMR spectroscopy}

${ }^{1} \mathrm{H}$ NMR (chloroform- $d$ ) $\delta(\mathrm{ppm}): 6.02(\mathrm{dd}, J=1.71,0.98 \mathrm{~Hz}, 1 \mathrm{H}), 5.47$ (quin, $J=1.59 \mathrm{~Hz}, 1$ H), 4.06 - $4.15(\mathrm{~m}, 2 \mathrm{H}), 1.86-1.89(\mathrm{~m}, 3 \mathrm{H}), 1.59$ - $1.69(\mathrm{~m}, 1 \mathrm{H}), 1.36$ - $1.54(\mathrm{~m}, 3 \mathrm{H}), 1.04$ - $1.28(\mathrm{~m}, 6 \mathrm{H}), 0.85(\mathrm{~d}, J=6.59 \mathrm{~Hz}, 3 \mathrm{H}), 0.80(\mathrm{~d}, J=6.59 \mathrm{~Hz}, 6 \mathrm{H})$
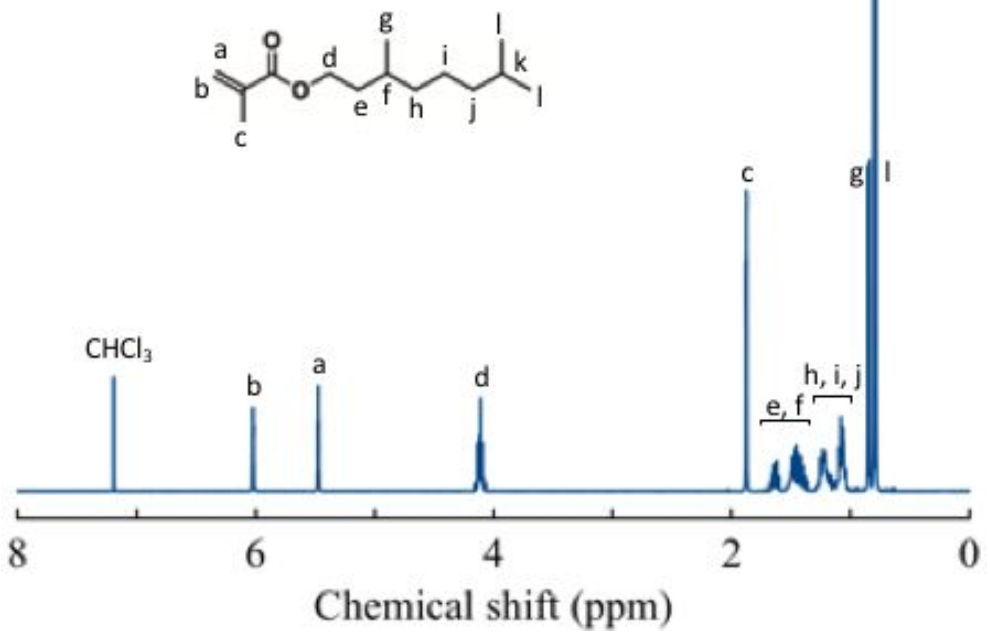

Figure S36 - ${ }^{1} \mathrm{H}$ NMR spectrum of 3,7-dimethyl-1-octanyl methacrylate in $\mathrm{CDCl}_{3}$

${ }^{13} \mathrm{C}$ APT NMR (chloroform- $d$ ) $\delta(\mathrm{ppm}): 166.36(\mathrm{C}), 130.40\left(\mathrm{CH}_{2}\right), 128.69(\mathrm{CH}), 63.18\left(\mathrm{CH}_{2}\right)$, $39.20\left(\mathrm{CH}_{2}\right), 37.14\left(\mathrm{CH}_{2}\right), 35.50\left(\mathrm{CH}_{2}\right), 29.88(\mathrm{CH}), 27.96(\mathrm{CH}), 24.61\left(\mathrm{CH}_{2}\right), 22.69\left(\mathrm{CH}_{3}\right)$, $22.60\left(\mathrm{CH}_{3}\right), 19.55\left(\mathrm{CH}_{3}\right)$

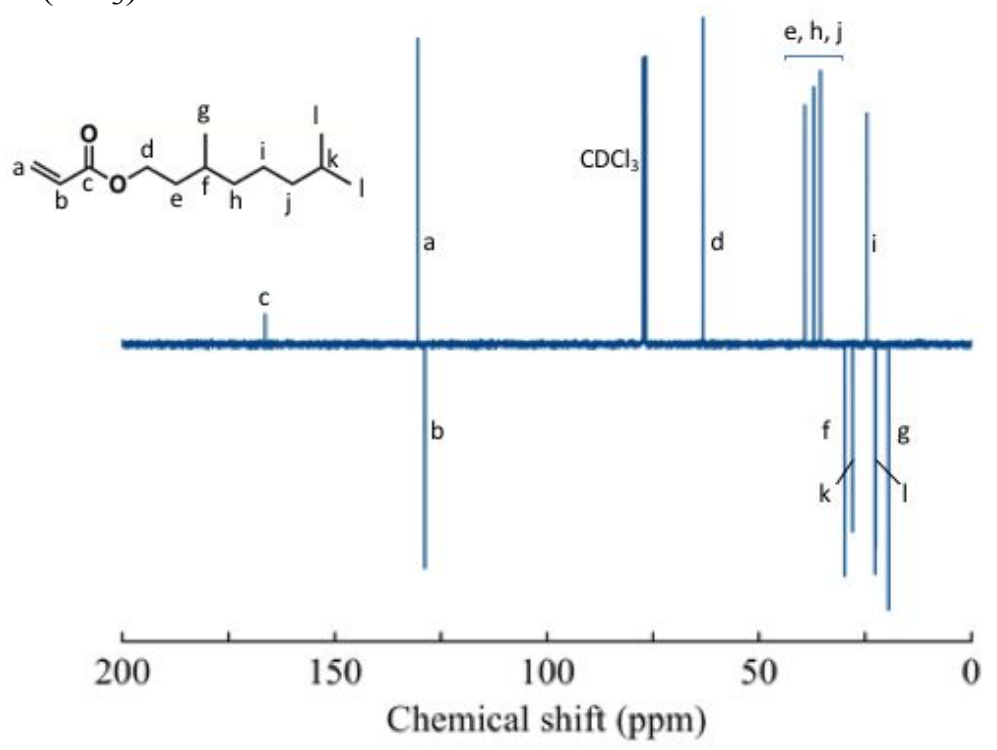

Figure $S 37-{ }^{13} \mathrm{C} A P T$ NMR spectrum of 3,7-dimethyl-1-octanyl methacrylate in $\mathrm{CDCl}_{3}$ 


\subsubsection{ESI-MS spectrometry}

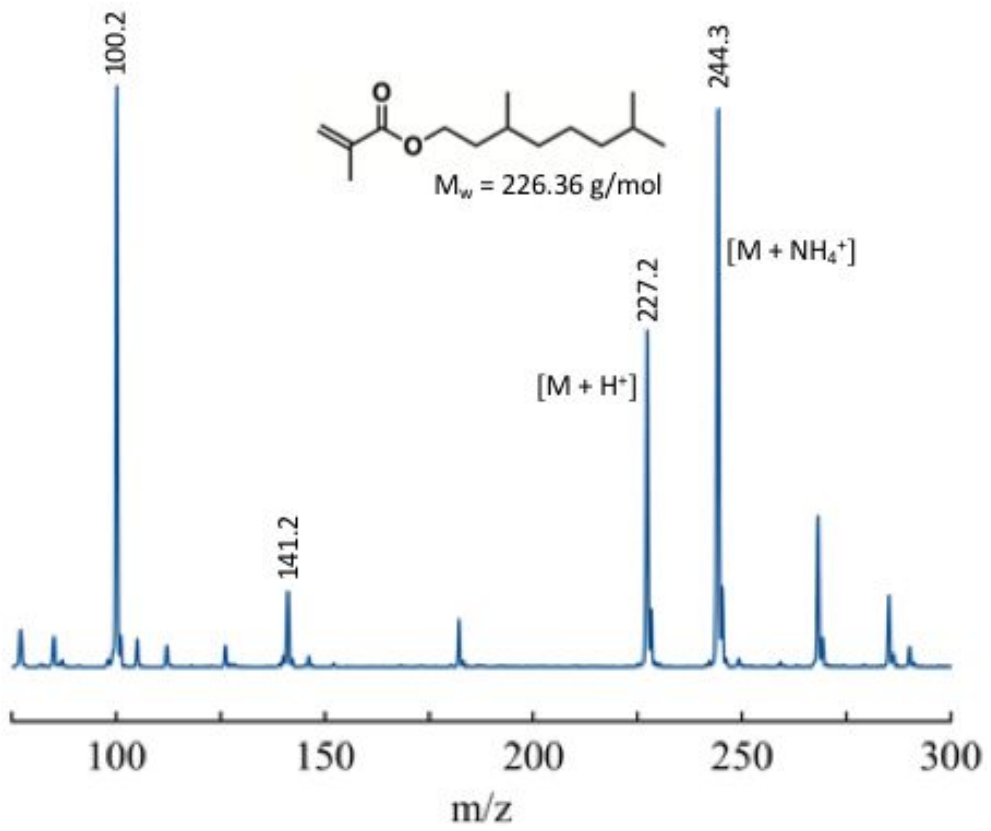

Figure S38 - ESI-MS spectrum of 3,7-dimethyl-1-octanyl methacrylate

*Peak at 100.2 is a system artefact.

1.6.3. GC-CI-MS analysis

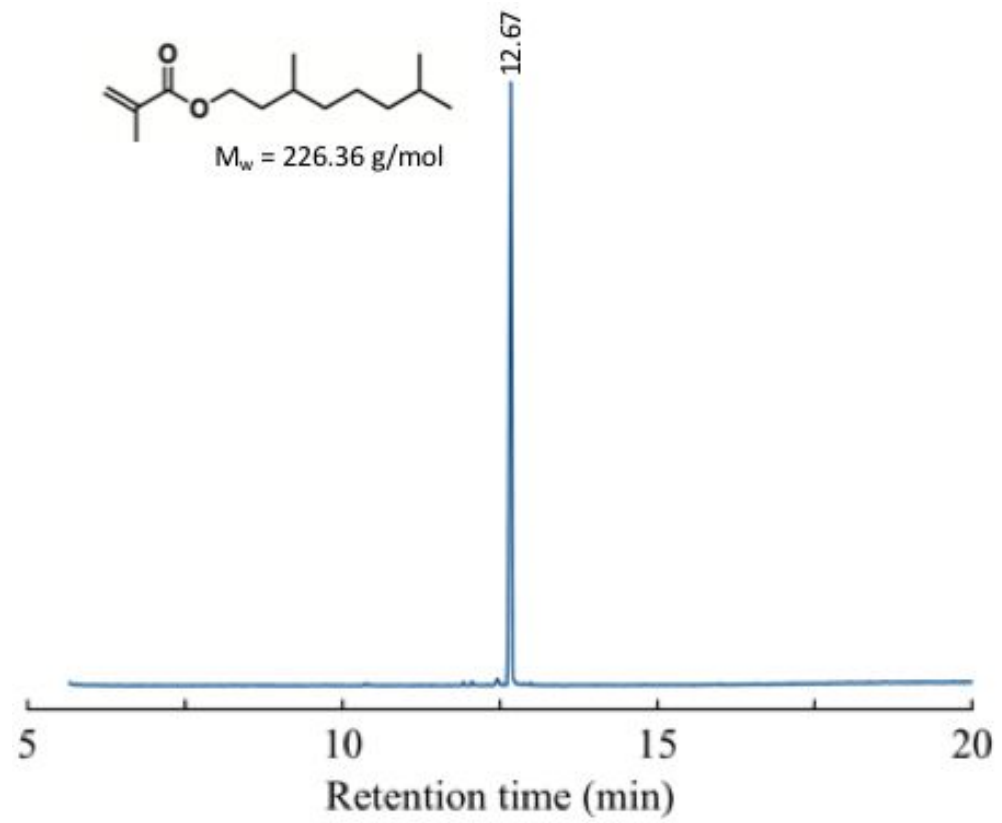

Figure S39 - GC spectrum obtained from the GC-CI-MS measurement of 3,7-dimethyl-1-octanyl methacrylate 


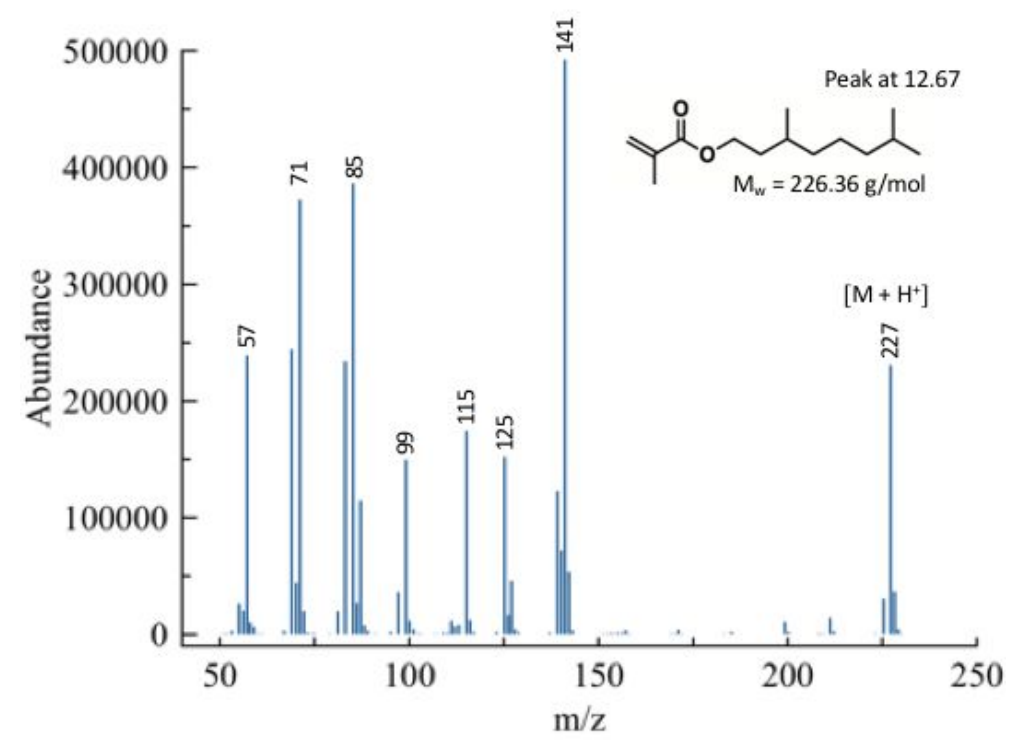

Figure S40 - CI-MS spectrum of 3,7-dimethyl-1-octanyl methacrylate, peak at $12.67 \mathrm{~min}$

\subsubsection{FTIR spectroscopy}

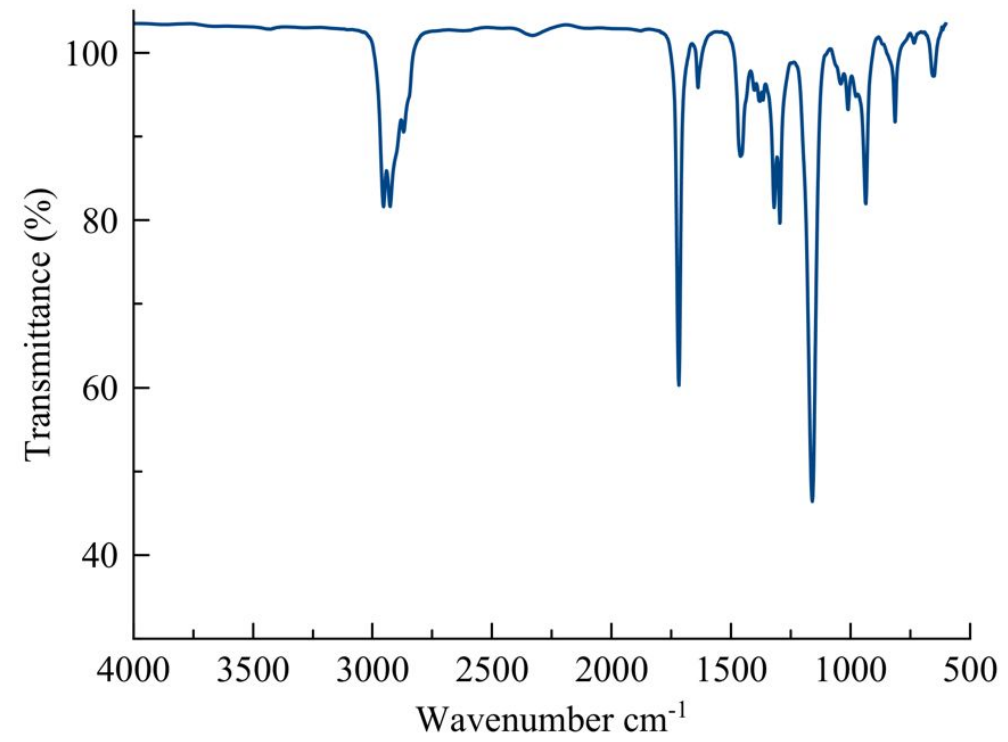

Figure S41 - FTIR spectrum of 3,7-dimethyl-1-octanyl methacrylate 


\subsection{Geranyl methacrylate}

\subsubsection{NMR spectroscopy}

${ }^{1} \mathrm{H}$ NMR (chloroform- $d$ ) $\delta$ (ppm): 6.02 - 6.05 (m, $1 \mathrm{H}$ ), 5.47 (quin, $J=1.62 \mathrm{~Hz}, 1 \mathrm{H}$ ), 5.28 - 5.33 (m, $1 \mathrm{H}), 4.99$ - $5.04(\mathrm{~m}, 1 \mathrm{H}), 4.58$ - $4.62(\mathrm{~m}, 2 \mathrm{H}), 1.95$ - 2.07 (m, $4 \mathrm{H}), 1.86$ - $1.89(\mathrm{~m}, 3 \mathrm{H})$, $1.65(\mathrm{~s}, 3 \mathrm{H}), 1.61(\mathrm{~d}, J=1.10 \mathrm{~Hz}, 3 \mathrm{H}), 1.53(\mathrm{~s}, 3 \mathrm{H})$
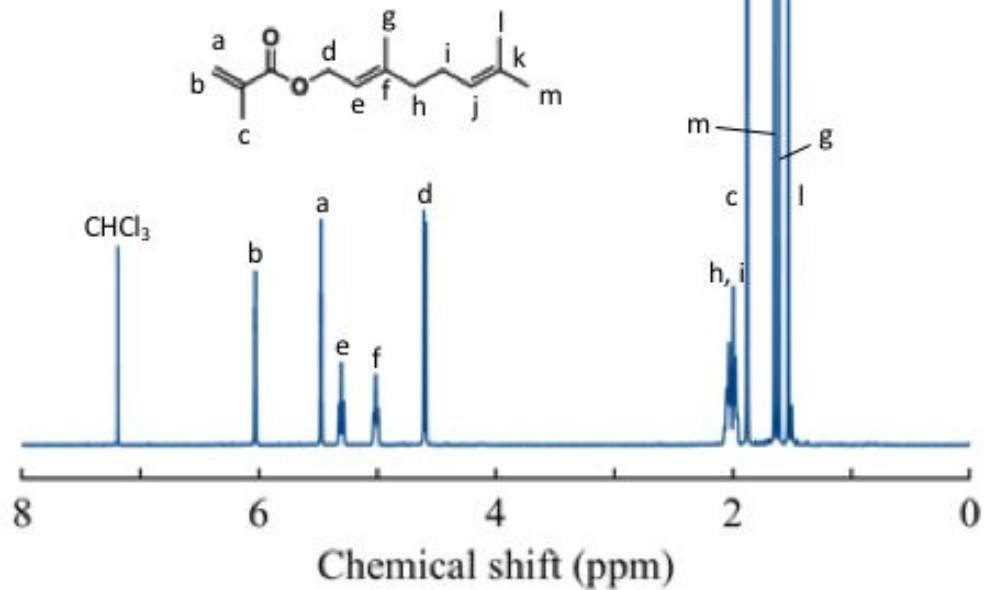

Figure S42 - ${ }^{1} \mathrm{H}$ NMR of geranyl methacrylate in $\mathrm{CDCl}_{3}$

${ }^{13} \mathrm{C}$ APT NMR (chloroform- $d$ ) $\delta$ (ppm): $167.54(\mathrm{C}), 142.02(\mathrm{C}), 136.57$ (C), $131.82(\mathrm{C}), 125.21$ $\left(\mathrm{CH}_{2}\right), 123.76(\mathrm{CH}), 118.47(\mathrm{CH}), 61.63\left(\mathrm{CH}_{2}\right), 39.53\left(\mathrm{CH}_{2}\right), 26.29\left(\mathrm{CH}_{2}\right), 25.68\left(\mathrm{CH}_{3}\right), 18.38$ $\left(\mathrm{CH}_{3}\right), 17.70\left(\mathrm{CH}_{3}\right), 16.50\left(\mathrm{CH}_{3}\right)$

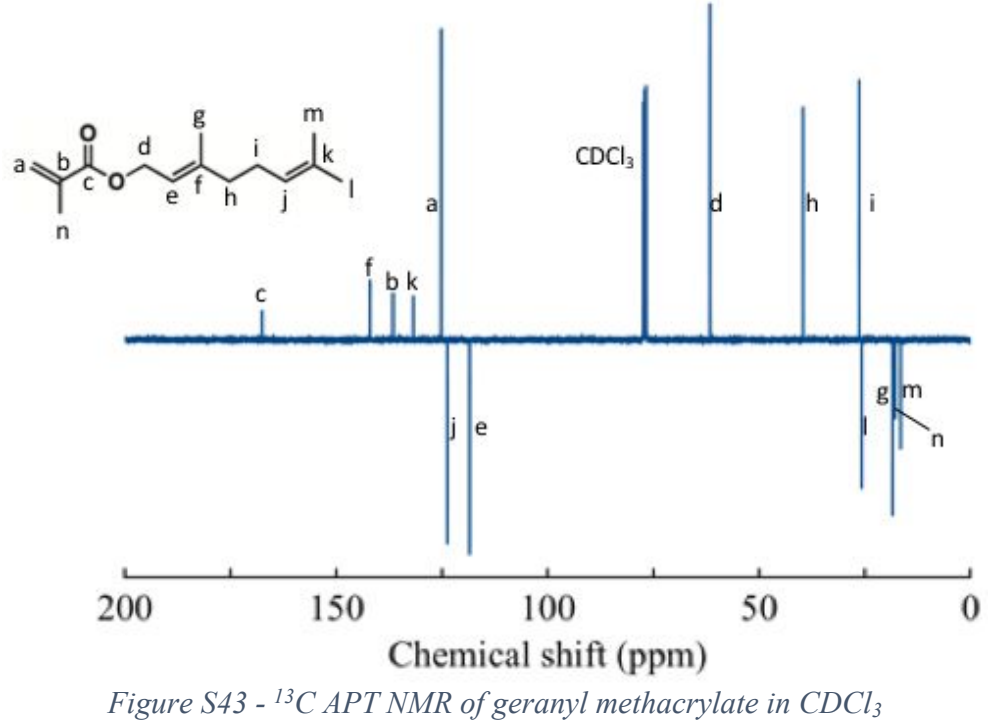




\subsubsection{ESI-MS spectrometry}

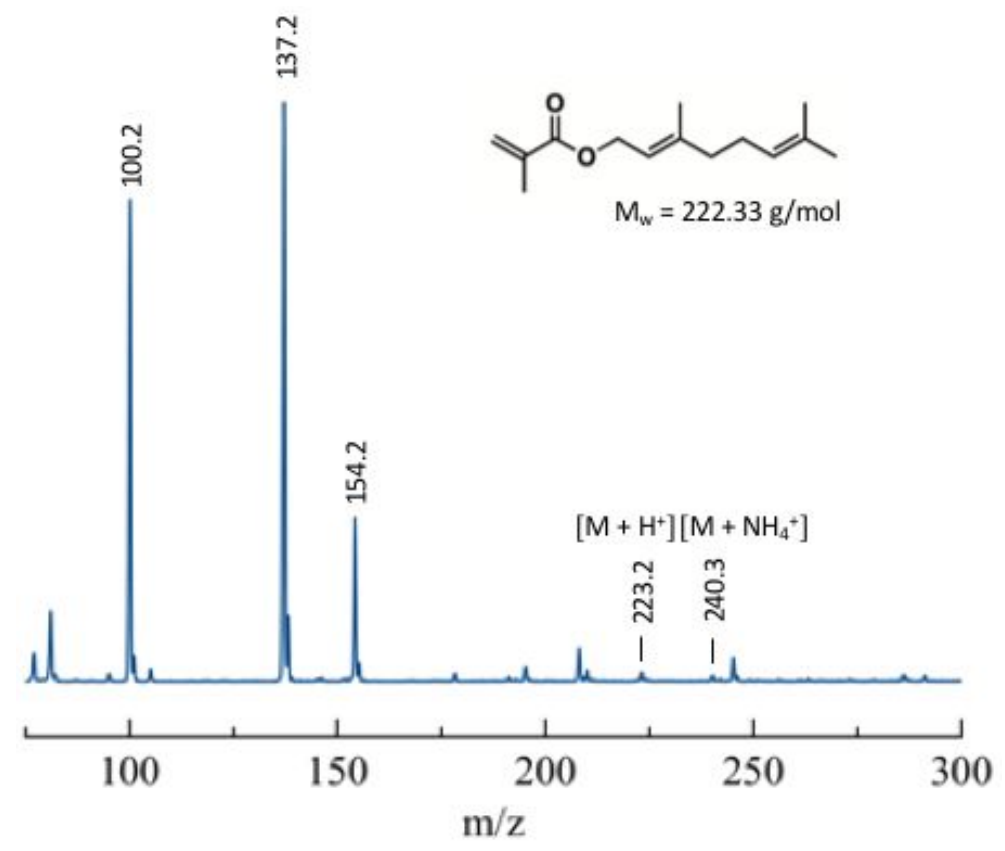

Figure S44 - ESI-MS spectrum of geranyl methacrylate

Low concentration of the geranyl methacrylate was observed in ESI-MS because of fragmentation of the compound. The peak at 100.2 is a system artefact.

1.7.3. GC-CI-MS analysis

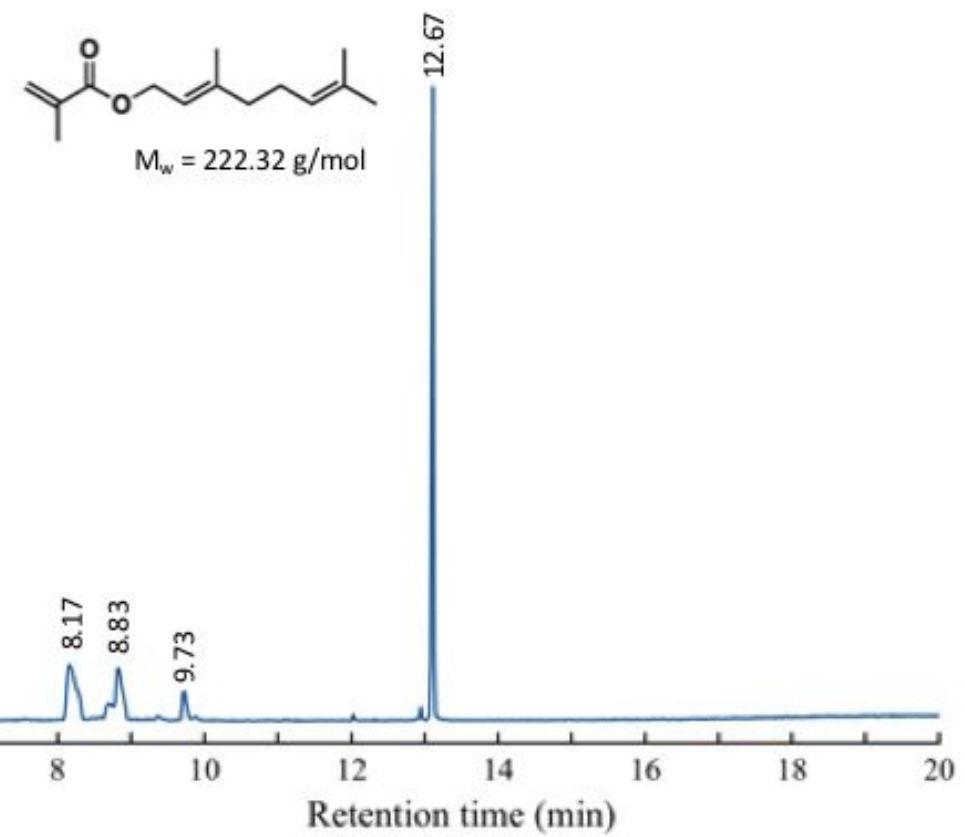

Figure S45 - GC spectrum obtained from the GC-CI-MS measurement of geranyl methacrylate 


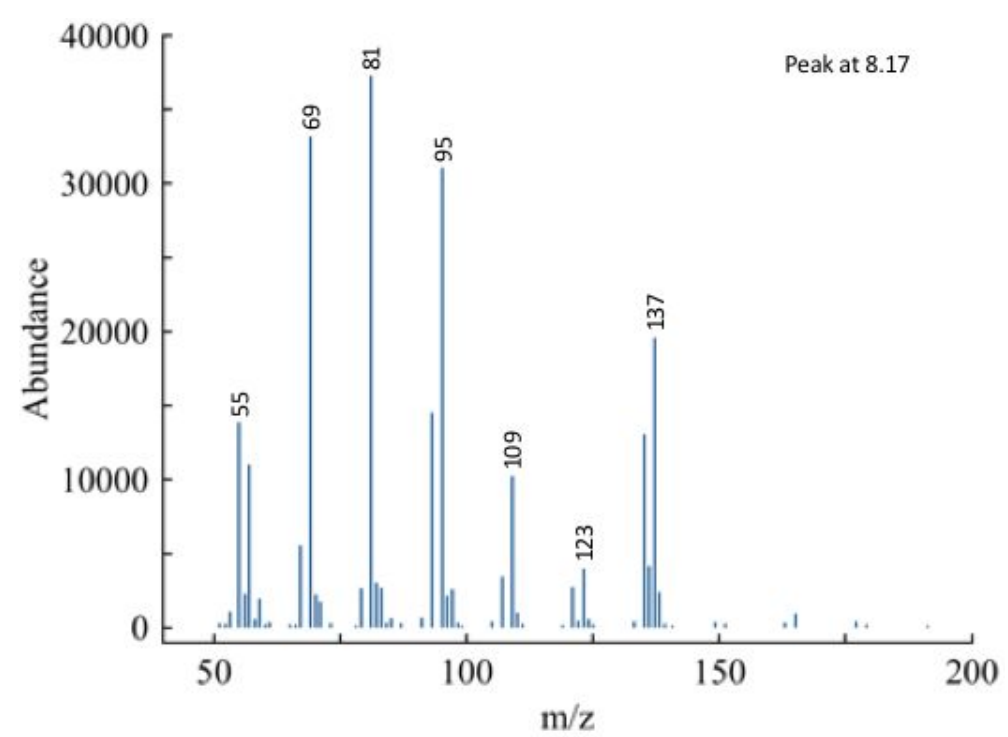

Figure S46 - CI-MS spectrum of a side product in the synthesis of geranyl methacrylate, peak at 8.17 min

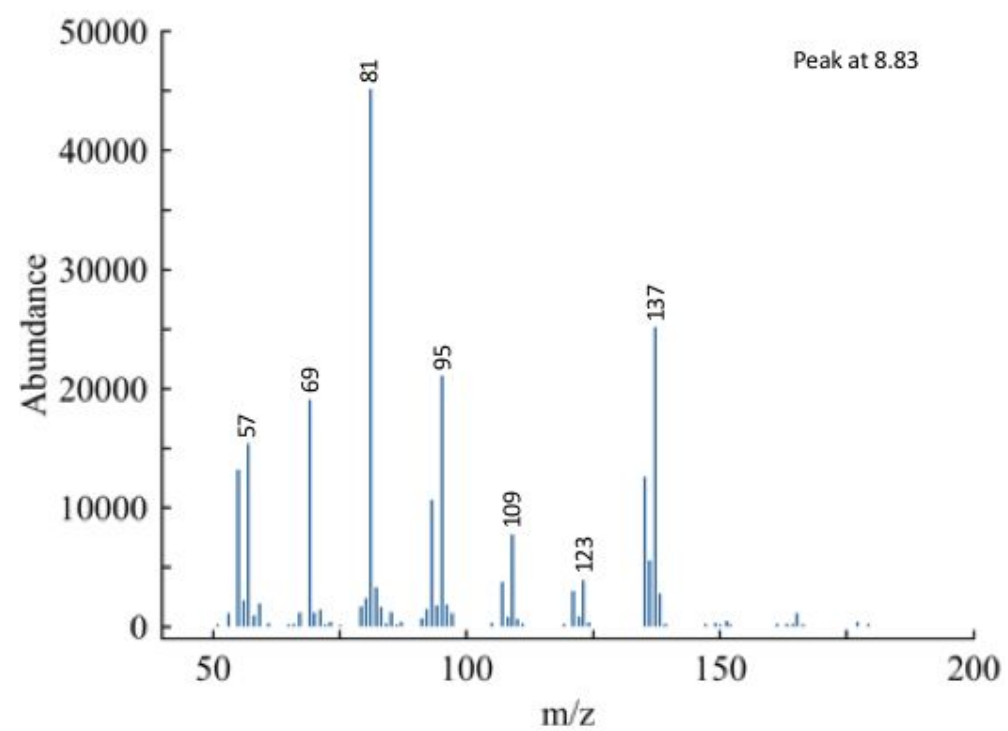

Figure S47 - CI-MS spectrum of a side product in the synthesis of geranyl methacrylate, peak at 8.83 min

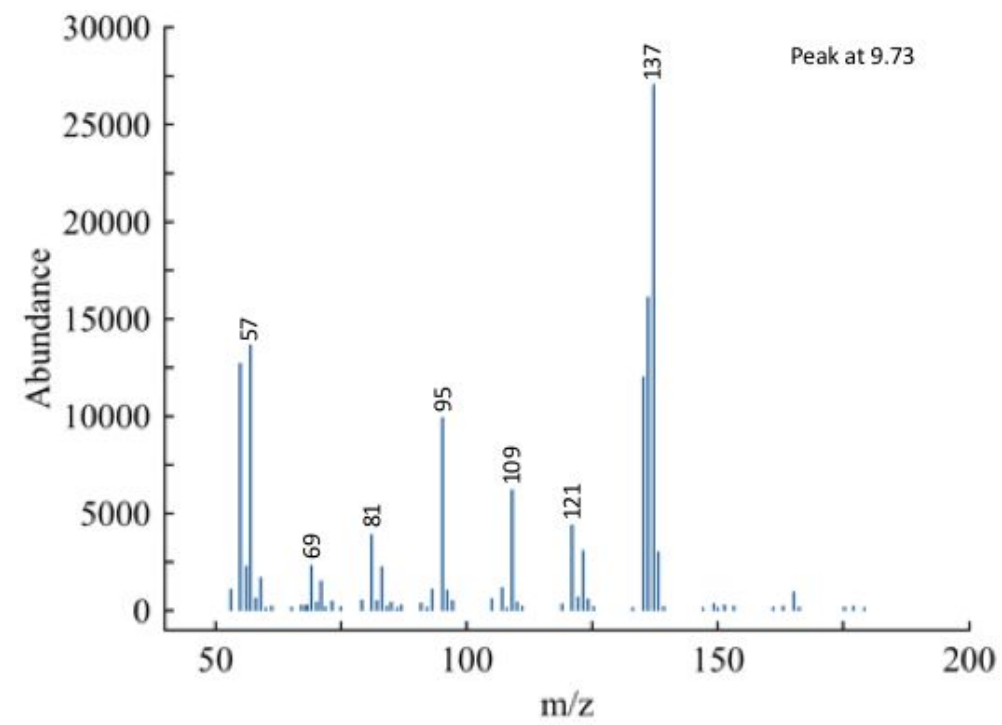

Figure S48 - CI-MS spectrum of a side product in the synthesis of geranyl methacrylate, peak at 9.73 min 


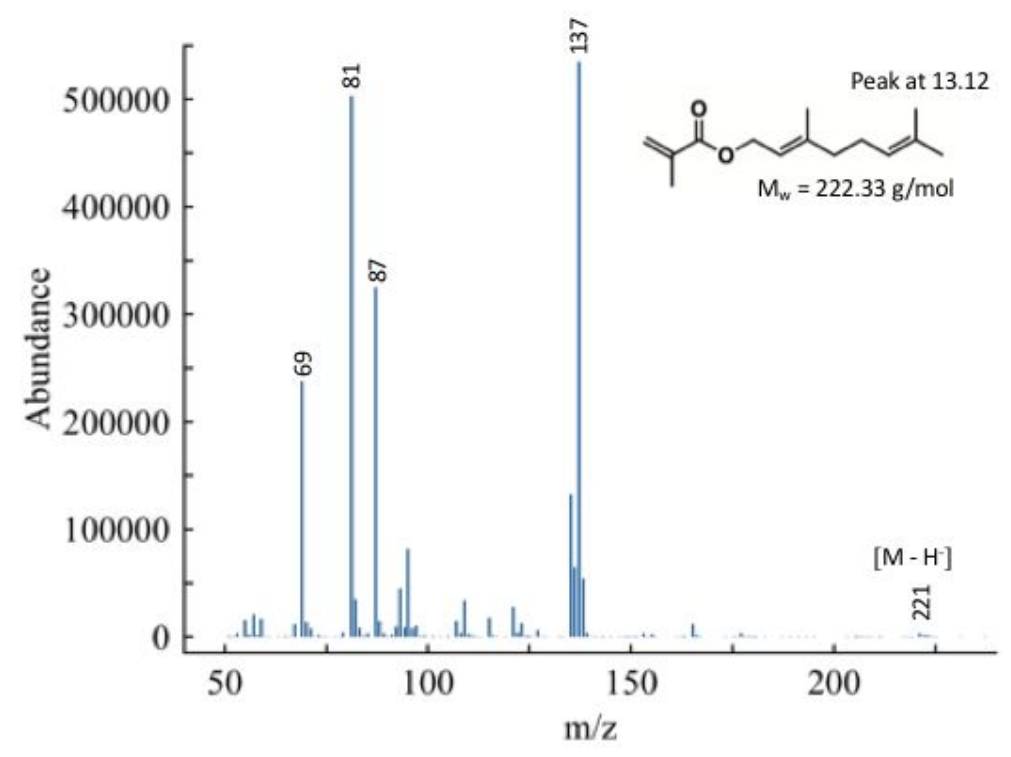

Figure S49 - CI-MS spectrum of geranyl methacrylate, peak at $13.12 \mathrm{~min}$

\subsubsection{FTIR spectroscopy}

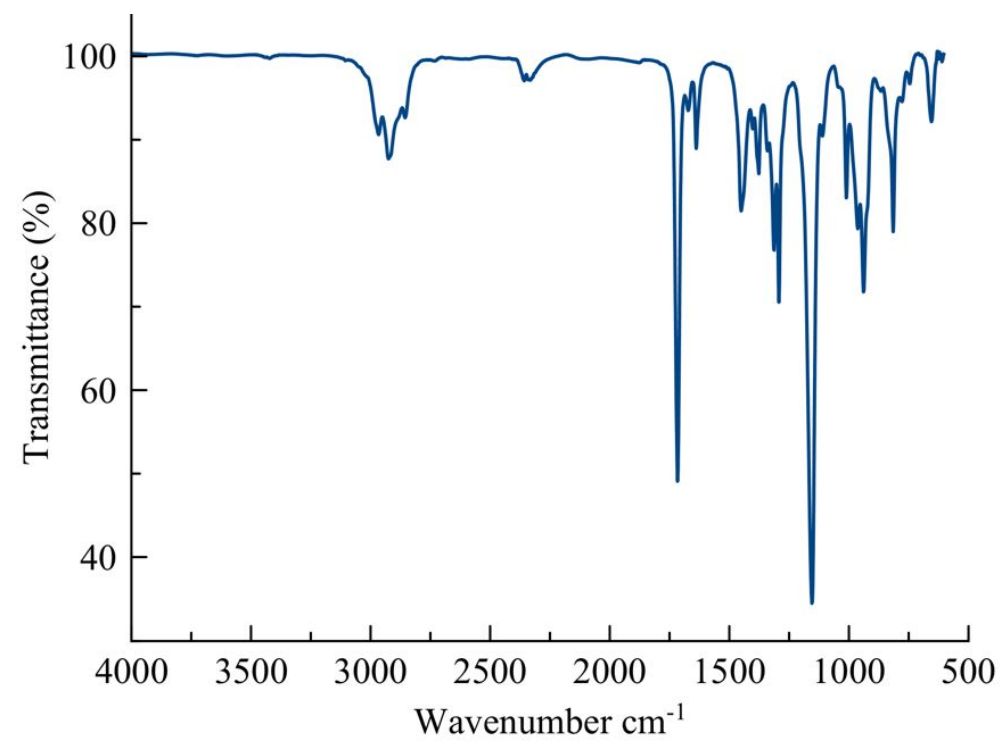

Figure S50 - FTIR spectrum of geranyl methacrylate 


\subsection{Menthyl methacrylate}

\subsubsection{NMR spectroscopy}

${ }^{1} \mathrm{H}$ NMR (chloroform- $d$ ) $\delta$ (ppm): 6.01 (dd, $J=1.71,0.98 \mathrm{~Hz}, 1 \mathrm{H}$ ), 5.45 (quin, $J=1.62 \mathrm{~Hz}, 1 \mathrm{H}$ ), $4.66(\mathrm{td}, J=10.86,4.39 \mathrm{~Hz}, 1 \mathrm{H}), 1.93-2.03(\mathrm{~m}, 1 \mathrm{H}), 1.87$ (dd, $J=1.59,0.98 \mathrm{~Hz}, 3 \mathrm{H}), 1.81$ $(\mathrm{dtd}, J=13.96,7.03,7.03,2.75 \mathrm{~Hz}, 1 \mathrm{H}), 1.59-1.66(\mathrm{~m}, 2 \mathrm{H}), 1.33-1.51(\mathrm{~m}, 2 \mathrm{H}), 0.88-1.09$ $(\mathrm{m}, 2 \mathrm{H}), 0.83(\mathrm{dd}, J=6.77,4.33 \mathrm{~Hz}, 7 \mathrm{H}), 0.66-0.72(\mathrm{~m}, 3 \mathrm{H})$
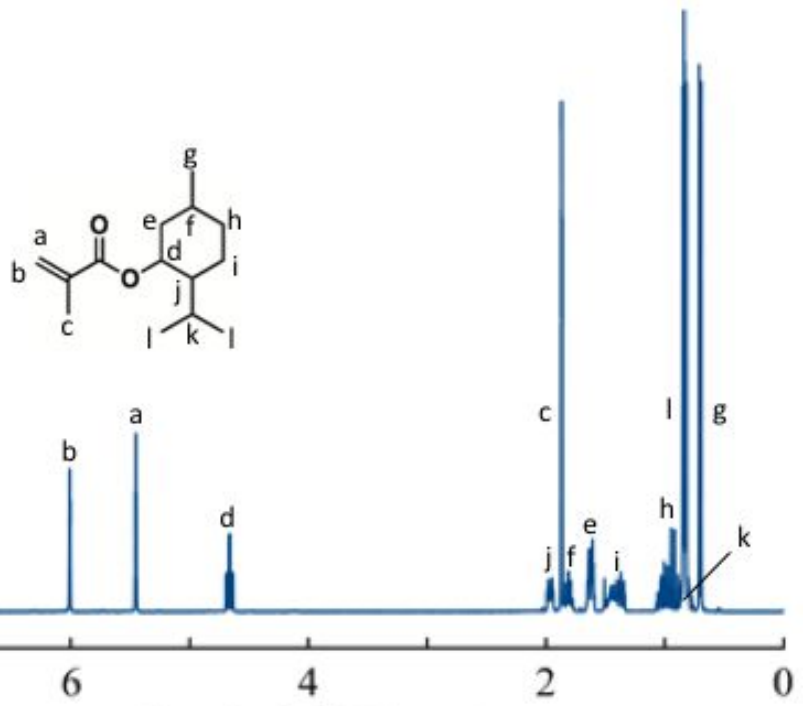

Chemical shift (ppm)

Figure $\mathrm{S} 51-{ }^{1} \mathrm{H} N \mathrm{NR}$ spectrum of menthyl methacrylate in $\mathrm{CDCl}_{3}$

${ }^{13} \mathrm{C}$ APT NMR (chloroform- $d$ ) $\delta(\mathrm{ppm}): 167.02(\mathrm{C}), 136.90(\mathrm{C}), 124.88\left(\mathrm{CH}_{2}\right), 74.47(\mathrm{CH})$, $47.16(\mathrm{CH}), 40.87\left(\mathrm{CH}_{2}\right), 34.31\left(\mathrm{CH}_{2}\right), 31.40(\mathrm{CH}), 26.45(\mathrm{CH}), 23.60\left(\mathrm{CH}_{2}\right), 22.04\left(\mathrm{CH}_{3}\right)$, $20.76\left(\mathrm{CH}_{3}\right), 18.40\left(\mathrm{CH}_{3}\right), 16.49\left(\mathrm{CH}_{3}\right)$

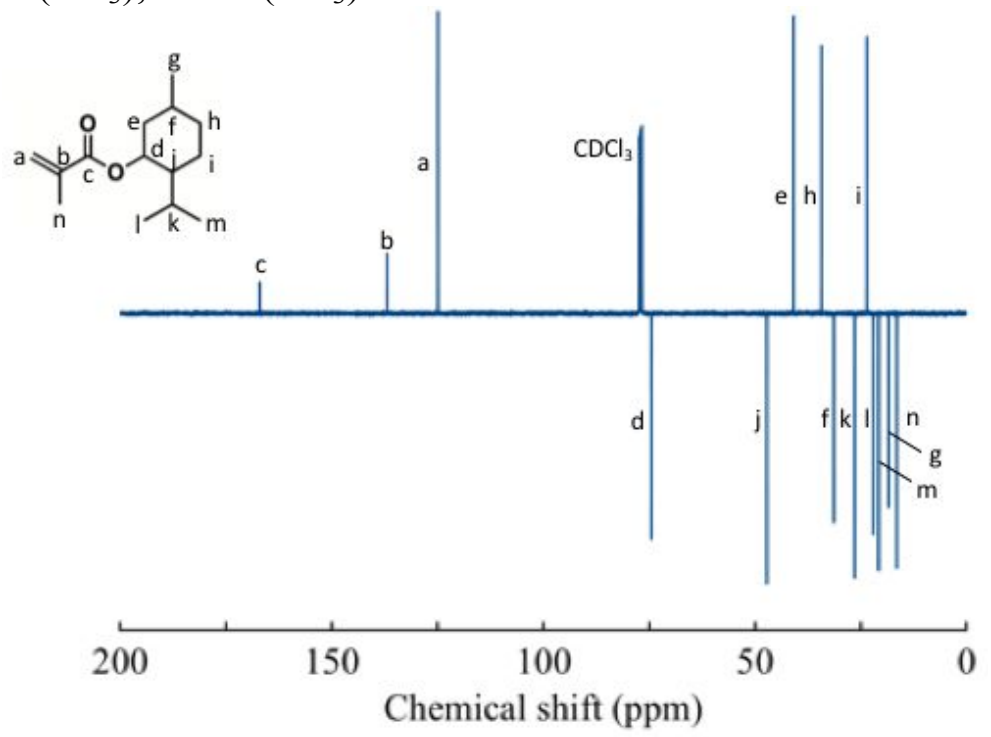

Figure S52 $-{ }^{13} \mathrm{C}$ APT NMR spectrum of menthyl methacrylate in $\mathrm{CDCl}_{3}$ 


\subsubsection{ESI-MS spectrometry}

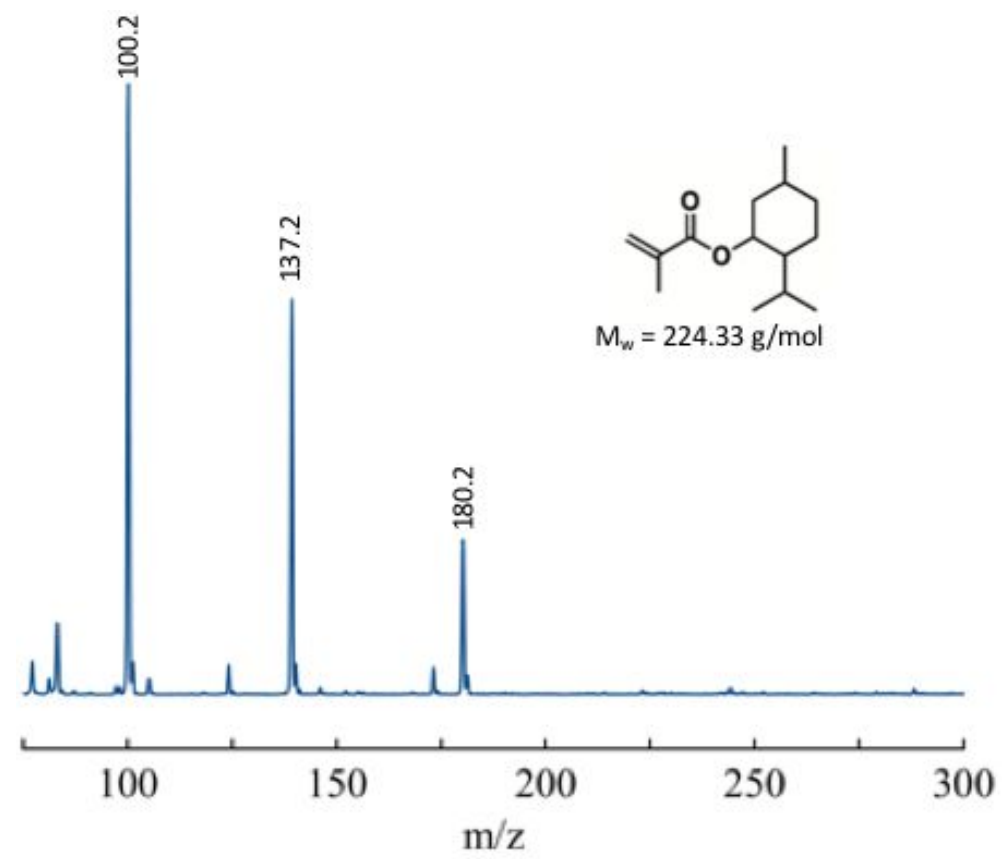

Figure S53 - ESI-MS spectrum of menthyl methacrylate

Menthyl acrylate was not visible in the ESI-MS spectrum but fragments of the compound were. The peak at 100.2 is a system artefact.

\subsubsection{GC-CI-MS analysis}

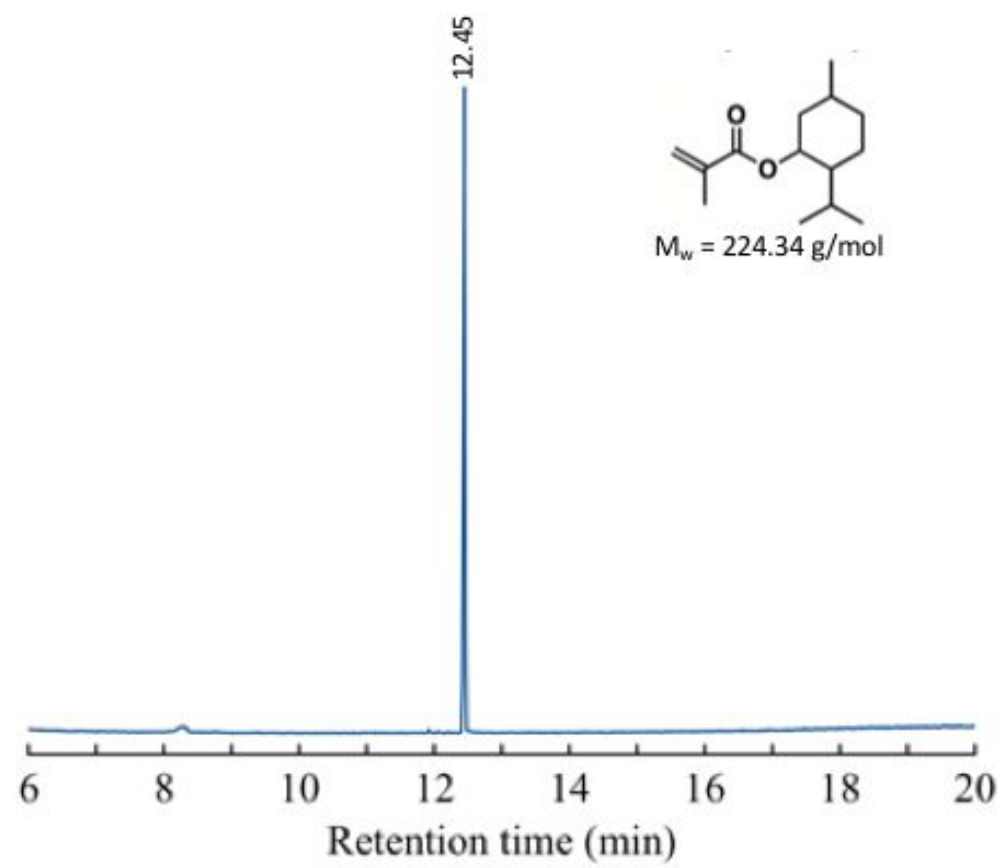

Figure S54 - GC spectrum obtained from the GC-CI-MS measurement of menthyl methacrylate 


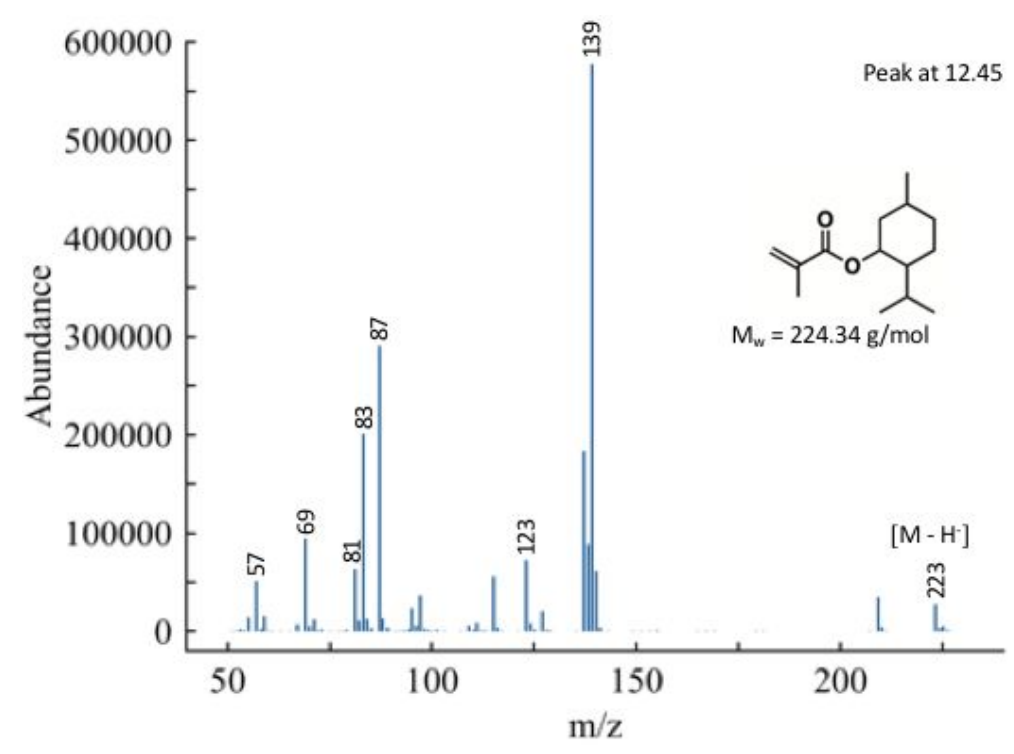

Figure S55 - CI-MS spectrum of menthyl methacrylate, peak at $12.45 \mathrm{~min}$

\subsubsection{FTIR spectroscopy}

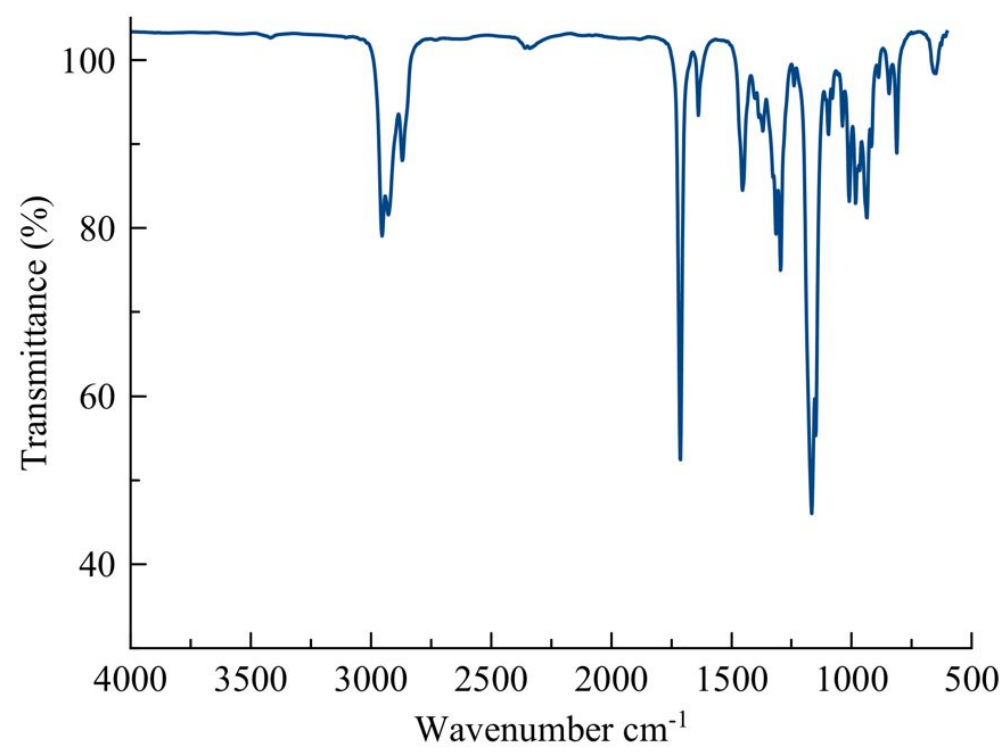

Figure S56 - FTIR spectrum of menthyl methacrylate 


\section{Detailed summary of the results obtained from the CHEM21 green metrics toolkit}

Table S1 - Detailed summary of the results obtained from the CHEM21 metric toolkit calculations

\begin{tabular}{|c|c|c|c|c|c|c|c|c|c|c|c|c|c|c|c|}
\hline \multirow{2}{*}{\multicolumn{2}{|c|}{ Acrylates }} & \multirow{2}{*}{ Catalyst } & \multirow{2}{*}{$\mathrm{T}\left({ }^{\circ} \mathrm{C}\right)$} & \multirow[b]{2}{*}{$t(h)$} & \multirow[b]{2}{*}{ Conv. (\%) } & \multirow[b]{2}{*}{ Yield $(\%)$} & \multirow[b]{2}{*}{ Select (\%) } & \multirow[b]{2}{*}{$\operatorname{AE}(\%)$} & \multirow[b]{2}{*}{ RME (\%) } & \multicolumn{4}{|c|}{ PMI (g/g) } & \multicolumn{2}{|r|}{ Flags } \\
\hline & & & & & & & & & & total & reaction & solvent & work-up & Solvent & E Cat $\mathrm{H}$-codes \\
\hline \multirow[t]{4}{*}{ Citronellyl acrylate } & Method A & - & RT & 16 & $>99$ & 71.4 & 71.4 & 85.2 & 56.4 & 38.4 & 14.6 & 34.0 & 23.8 & $\mathrm{DCM}, \mathrm{THF}, \mathrm{H}_{2} \mathrm{O}$ & Н330, Н311, Н331, Н351, Н371 \\
\hline & Method B & pTSOH & 130 & 24 & 92 & 81.7 & 88.8 & 86.8 & 41.4 & 2.5 & 2.5 & 0 & 0 & - & H331, H373, H412 \\
\hline & Method D & $\mathrm{pTSOH}$ & 130 & 6 & 94 & 73.2 & 77.9 & 92.1 & 62.5 & 3.1 & 3.1 & 1.5 & 0 & Toluene & $\mathrm{H} 400 \mathrm{O}, \mathrm{H} 361, \mathrm{H} 373, \mathrm{H} 412$ \\
\hline & Method E & $\mathrm{pTSOH}$ & 130 & 6 & 97 & 72.8 & 75 & 92.1 & 62.2 & 2.6 & 2.6 & 0 & 0 & - & $\mathrm{H} 400, \mathrm{H} 373, \mathrm{H} 412$ \\
\hline \multirow[t]{4}{*}{ 3,7-Dimethyl-1-octanyl acrylate } & Method A & - & RT & 16 & $>99$ & 71.5 & 71.5 & 85.3 & 56.9 & 41.9 & 14.6 & 37.6 & 27.3 & $\mathrm{DCM}, \mathrm{THF}, \mathrm{H}_{2} \mathrm{O}$ & H330, Н411, Н311, H331, H351, H371 \\
\hline & Method B & $\mathrm{pTSOH}$ & 130 & 24 & 83 & 78.2 & 94.3 & 86.9 & 39.9 & 2.6 & 2.6 & 0 & 0 & - & $\mathrm{H} 411, \mathrm{H} 373, \mathrm{H} 412$ \\
\hline & Method D & pTSOH & 130 & 6 & 95 & 81.1 & 85.4 & 92.2 & 69.3 & 2.8 & 2.8 & 1.3 & 0 & Toluene & $\mathrm{H} 400, \mathrm{H} 411, \mathrm{H} 361, \mathrm{H} 373, \mathrm{H} 412$ \\
\hline & Method E & $\mathrm{pTSOH}$ & 130 & 6 & 97 & 80.2 & 82.7 & 93 & 69.2 & 2.3 & 2.3 & 0 & 0 & - & $\mathrm{H} 400 \mathrm{O}, \mathrm{H} 411, \mathrm{H} 373, \mathrm{H} 412$ \\
\hline \multirow[t]{2}{*}{ Geranyl acrylate } & Method A & & RT & 16 & $>99$ & 41.8 & 41.8 & 85.1 & 33.2 & 147.7 & 28.4 & 135.9 & 119.3 & $\mathrm{DCM}, \mathrm{THF}, \mathrm{H}_{2} \mathrm{O}$ & H330, Н311, Н331, Н351, Н371 \\
\hline & Method C & Lipase & 40 & 16 & $>99$ & 99 & 99 & 86.7 & 63.1 & 5.3 & 3.1 & 2.2 & 2.2 & мTBE & $\mathrm{H} 331, \mathrm{H} 412$ \\
\hline \multirow[t]{3}{*}{ Menthyl acrylate } & Method A & - & RT & 16 & 95 & 62.7 & 66 & 85.2 & 49.8 & 64.9 & 21.8 & 59.3 & 43.1 & $\mathrm{DCM}, \mathrm{THF}, \mathrm{H}_{2} \mathrm{O}$ & H330, H311, H331, H351, H371 \\
\hline & Method B & pTSOH & 130 & 24 & 75 & 67 & 89.3 & 86.8 & 34 & 3 & 3 & 0 & 0 & & $\mathrm{H} 331, \mathrm{H} 373, \mathrm{H} 412$ \\
\hline & Method E & $\mathrm{pTSOH}$ & 130 & 6 & 94 & 60.3 & 64.2 & 92.1 & 51.5 & 3.1 & 3.1 & 0 & 0 & - & $\mathrm{H} 400, \mathrm{H} 373, \mathrm{H} 412$ \\
\hline 2-Ethylhexyl acrylate & Method E & $\mathrm{pTSOH}$ & 130 & 6 & 95 & 79 & $\sim 100$ & 91 & 66 & 2.3 & 2.3 & 0 & 0 & - & $\mathrm{H} 400 \mathrm{O}, \mathrm{H} 373, \mathrm{H} 412$ \\
\hline \multicolumn{16}{|l|}{ Methacrylates } \\
\hline \multirow{3}{*}{ Citronellyl methacrylate } & Method A & - & RT & 16 & $>99$ & 39.2 & 39.2 & 86 & 31.2 & 94.4 & 24.4 & 85.4 & 70 & $\mathrm{DCM}, \mathrm{THF}, \mathrm{H}_{2} \mathrm{O}$ & $\mathrm{H} 3330, \mathrm{H} 311, \mathrm{H} 331, \mathrm{H} 351, \mathrm{H} 371, \mathrm{H} 412$ \\
\hline & Method B & pTSOH & 130 & 24 & 90 & 69.1 & 76.8 & 87.5 & 33.9 & 3 & 3 & 0 & 0 & - & $\mathrm{H} 373, \mathrm{H} 412$ \\
\hline & Method E & $\mathrm{pTSOH}$ & 130 & 6 & 95 & 71.5 & 75.2 & 92.6 & 60.8 & 2.6 & 2.6 & 0 & 0 & - & $\mathrm{H} 311, \mathrm{H} 373, \mathrm{H} 412$ \\
\hline \multirow[t]{3}{*}{ 3,7-Dimethyl-1-octanyl methacrylate } & Method A & - & RT & 16 & 94 & 55.3 & 58.8 & 86.1 & 44.1 & 78.5 & 26.1 & 72.2 & 52.4 & $\mathrm{DCM}, \mathrm{THF}, \mathrm{H}_{2} \mathrm{O}$ & H330, Н411, Н311, Н331, Н351, Н371, Н412 \\
\hline & Method B & PTSOH & 130 & 24 & 85 & 73.3 & 86.2 & 87.6 & 36.2 & 2.8 & 2.8 & 0 & 0 & - & H411, H373, H412 \\
\hline & Method E & $\mathrm{pTSOH}$ & 130 & 6 & 97 & $\sim 100$ & $\sim 100$ & 87.6 & 80 & 1.9 & 1.9 & 0 & 0 & - & $\mathrm{H} 411, \mathrm{H} 311, \mathrm{H} 373, \mathrm{H} 412$ \\
\hline \multirow[t]{2}{*}{ Geranyl methacrylate } & Method A & - & RT & 16 & 97 & 57.6 & 59.4 & 85.9 & 45.8 & 76.8 & 25.5 & 70.6 & 51.3 & $\mathrm{DCM}, \mathrm{THF}, \mathrm{H}_{2} \mathrm{O}$ & H330, H311, $, 3331, \mathrm{H3} 31, \mathrm{H} 371, \mathrm{H} 412$ \\
\hline & Method C & Lipase & 40 & 48 & 45 & 27.3 & 69.4 & 87.4 & 17.1 & 18.5 & 10.9 & 7.5 & 7.5 & MTBE & $\mathrm{H} 331, \mathrm{H} 412$ \\
\hline \multirow[t]{2}{*}{ Menthyl methacrylate } & Method A & - & RT & 16 & 93 & 71.8 & 77.3 & 86 & 57.2 & 45.5 & 9.9 & 40.6 & 35.6 & $\mathrm{DCM}, \mathrm{THF}, \mathrm{H}_{2} \mathrm{O}$ & H330, Н311, Н331, H351, H371, H412 \\
\hline & Method E & pTSOH & 130 & 24 & 96 & 91.9 & 95.7 & 87.5 & 73.3 & 2.1 & 2.1 & 0 & 0 & - & $\mathrm{H} 311, \mathrm{H} 373, \mathrm{H} 412$ \\
\hline
\end{tabular}

Select: Selectivity (\%), AE: Atom Efficiency (\%), RME: Reaction Mass Efficiency (\%), PMI: Process Mass Intensity, E: Energy consumption, Cat: use of catalyst, H-codes: Hazard codes 
3. An example of the calculation of the Zero Pass for citronellyl acrylate using Method A

\begin{tabular}{|c|c|c|c|c|c|c|c|c|c|c|c|}
\hline $\begin{array}{c}\text { Reactant (Limiting Reactant } \\
\text { First) }\end{array}$ & $\begin{array}{c}\text { Mass } \\
\text { (g) }\end{array}$ & MW & Mol & Catalyst & Mass (g) & Reagent & Mass (g) & Reaction solvent & $\begin{array}{l}\text { Volume } \\
\left(\mathrm{cm}^{3}\right)\end{array}$ & $\begin{array}{l}\text { Density } \\
\left(\mathrm{g} \mathrm{ml}^{-1}\right)\end{array}$ & Mass (g) \\
\hline Citronellol & 20,00 & 156,27 & 0,1280 & & & Triethylamine & 24,90 & Dichloromethane & 250,00 & 0,89 & 222,25 \\
\hline Acryloyl Chloride & 14,08 & 90,51 & 0,1556 & & & & & & & & 0,00 \\
\hline Total & 34,08 & 246,78 & & & 0,00 & & 24,90 & & & & 222,25 \\
\hline
\end{tabular}

Flag

$$
\begin{aligned}
& A E=\frac{\text { molecular weight of product }}{\text { total molecualr weight of reactants }} \times 100 \\
& R M E=\frac{\text { mass of isolated product }}{\text { total mass of reactants }} \times 100
\end{aligned}
$$

$\begin{array}{lr}\begin{array}{l}\text { Yield (\%) } \\ \text { Conversion } \\ (\%)\end{array} & 71,4 \\ \text { Selectivity (\%) } & 100,0 \\ & 71,4 \\ \text { AE (\%) } & 85,2 \\ \text { RME (\%) } & 56,4\end{array}$

Solvents (Zero Pass)

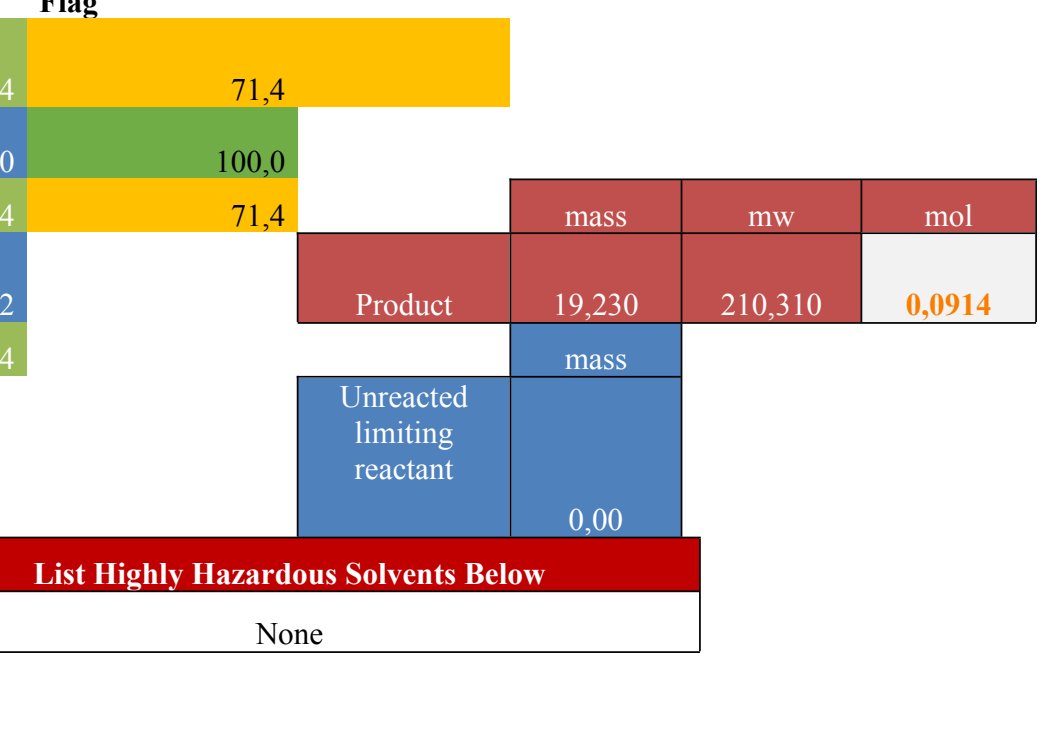




\begin{tabular}{|c|c|c|}
\hline \multicolumn{2}{|c|}{ List substances plus the red flagged H-codes below } \\
\hline Highly explosive & $\mathrm{H} 200, \mathrm{H} 201, \mathrm{H} 202, \mathrm{H} 203$ & \\
\hline Explosive thermal runaway & $\mathrm{H} 240$ & \\
\hline Fatally toxic & $\mathrm{H} 300, \mathrm{H} 310, \mathrm{H} 330$ & \\
\hline Mutagenic & $\mathrm{H} 350$ & \\
\hline Repro-toxic & $\mathrm{H} 360$ & \\
\hline Serious environmental implications & $\mathrm{H} 420$ & \\
\hline
\end{tabular}


4. An example of the calculation of the First Pass for citronellyl acrylate using Method A

Yield, AE, RME, MI/PMI and OE

\begin{tabular}{|c|c|c|c|c|c|c|c|c|c|c|c|}
\hline $\begin{array}{l}\text { Reactant (Limiting } \\
\text { Reactant First) }\end{array}$ & Mass (g) & MW & Mol & Catalyst & Mass (g) & Reagent & Mass (g) & Reaction solvent & Volume $\left(\mathrm{cm}^{3}\right)$ & $\begin{array}{c}\underset{\text { Density }}{\left.\mathrm{ml}^{-1}\right)} \\
\text { (g }\end{array}$ & Mass (g) \\
\hline Citronellol & 20,00 & 156,27 & 0,13 & & & Triethylamine & 24,90 & Dichloromethane & 250,00 & 0,89 & 222,25 \\
\hline Acryloyl Chloride & 14,08 & 90,51 & 0,16 & & & & & & & & 0,00 \\
\hline Total & 34,08 & 246,78 & & & 0,00 & & 24,90 & & & & 222,25 \\
\hline
\end{tabular}

\begin{tabular}{|c|c|c|c|c|c|}
\hline Work up chemical & Mass (g) & Workup solvent & $\begin{array}{c}\text { Volume } \\
\left(\mathrm{cm}^{3}\right)\end{array}$ & $\begin{array}{c}\text { Density } \\
\left(\mathrm{g} \mathrm{ml}^{-1}\right)\end{array}$ & Mass $(g)$ \\
\hline $\mathrm{HCl}$ & 5,47 & Water & 300,00 & 1,00 & 299,10 \\
\hline $\mathrm{NaHCO} 3$ & 20,00 & Dichloromethane & 100,00 & 1,33 & 132,50 \\
\hline Total & 25,47 & & & & 431,60 \\
\hline
\end{tabular}

\begin{tabular}{|c|c|c|}
\hline & & Flag \\
\hline Yield (\%) & 71,4 & 71,4 \\
\hline Conversion (\%) & 100,0 & 100,0 \\
\hline Selectivity (\%) & 71,4 & 71,4 \\
\hline $\mathrm{AE}(\%)$ & 85,2 & \\
\hline RME (\%) & 56,4 & \\
\hline PMI $\left(\mathrm{g} \mathrm{g}^{-1}\right)$ & 38,4 & \\
\hline PMI reaction $\left(\mathrm{g} \mathrm{g}^{-1}\right)$ & 14,6 & \\
\hline PMI solvent $\left(\mathrm{g} \mathrm{g}^{-1}\right)$ & 34,0 & \\
\hline PMI workup $\left(\mathrm{g} \mathrm{g}^{-1}\right)$ & 23,8 & \\
\hline
\end{tabular}

\begin{tabular}{|c|c|c|c|}
\cline { 2 - 3 } \multicolumn{1}{c|}{} & Mass $(\mathrm{g})$ & MW $(\mathrm{g}$ mmol & Mol \\
\hline Product & 19,230 & 210,310 & 0,0914 \\
\hline Unreacted limiting reactant & Mass $(\mathrm{g})$ & \multicolumn{2}{c}{} \\
\hline
\end{tabular}


Solvents (First Pass)

\begin{tabular}{|c|c|c|}
\hline Solvents (First Pass) & & List solvents below \\
\hline Preferred solvents & $\begin{array}{l}\text { water, } \mathrm{EtOH}, \mathrm{nBuOH}, \mathrm{AcOipr}, \mathrm{AcOnBu}, \mathrm{PhOMe}, \mathrm{MeOH}, \mathrm{tBuOH} \text {, } \\
\mathrm{BnOH} \text {, ethylene glycol, acetone, MEK, MIBK, AcOEt, sulfolane }\end{array}$ & water \\
\hline $\begin{array}{l}\text { Problematic solvents: (acceptable only } \\
\text { if substitution does not offer advantages) }\end{array}$ & $\begin{array}{l}\text { DMSO, cyclohexanone, DMPU, AcOH, Ac2O, Acetonitrile, } \\
\text { AcOMe, THF, heptane, Me-cyclohexane, toluene, xylene, MTBE, } \\
\text { cyclohexane, chlorobenzene, formic acid, pyridine, Me-THF }\end{array}$ & \\
\hline $\begin{array}{l}\text { Hazardous solvents: These solvents } \\
\text { have significant health and/or safety } \\
\text { concerns. }\end{array}$ & $\begin{array}{c}\text { dioxane, pentane, TEA, diisopropyl ether, DME, DCM, DMF, } \\
\text { DMA, NMP, methoxyethanol, hexane }\end{array}$ & DCM \\
\hline $\begin{array}{l}\text { Highly hazardous solvents: The } \\
\text { solvents which are agreed not to be used, } \\
\text { even in screening }\end{array}$ & $\mathrm{Et}_{2} \mathrm{O}$, Benzene, $\mathrm{CCl}_{4}$, chloroform, DCE, nitromethane, $\mathrm{CS}_{2}, \mathrm{HMPA}$ & \\
\hline
\end{tabular}

\begin{tabular}{|c|c|c|}
\hline \multicolumn{2}{|l|}{ Catalyst/enzyme (First Pass) } & Tick \\
\hline $\begin{array}{l}\text { Catalyst or enzyme used, or reaction takes place without } \\
\text { any catalyst/reagents. }\end{array}$ & $\begin{array}{l}\text { Green } \\
\text { Flag }\end{array}$ & \\
\hline Use of stoichiometric quantities of reagents & $\begin{array}{l}\text { Amber } \\
\text { Flag }\end{array}$ & \\
\hline Use of reagents in excess & Red Flag & $\mathrm{x}$ \\
\hline
\end{tabular}

\begin{tabular}{|c|c|c|}
\cline { 3 - 3 } \multicolumn{2}{|c|}{ Tick } \\
\hline Facile recovery of catalyst/enzyme & Green Flag & \\
\hline catalyst/enzyme not recovered & Amber Flag & \\
\hline
\end{tabular}

Critical elements
\begin{tabular}{|c|c|c|} 
Supply remaining & Flag colour & $\begin{array}{c}\text { Note } \\
\text { element }\end{array}$ \\
\hline $5-50$ years & Red Flag & \\
\hline $50-500$ years & Amber Flag & \\
\hline+500 years & Green Flag & All \\
\hline
\end{tabular}

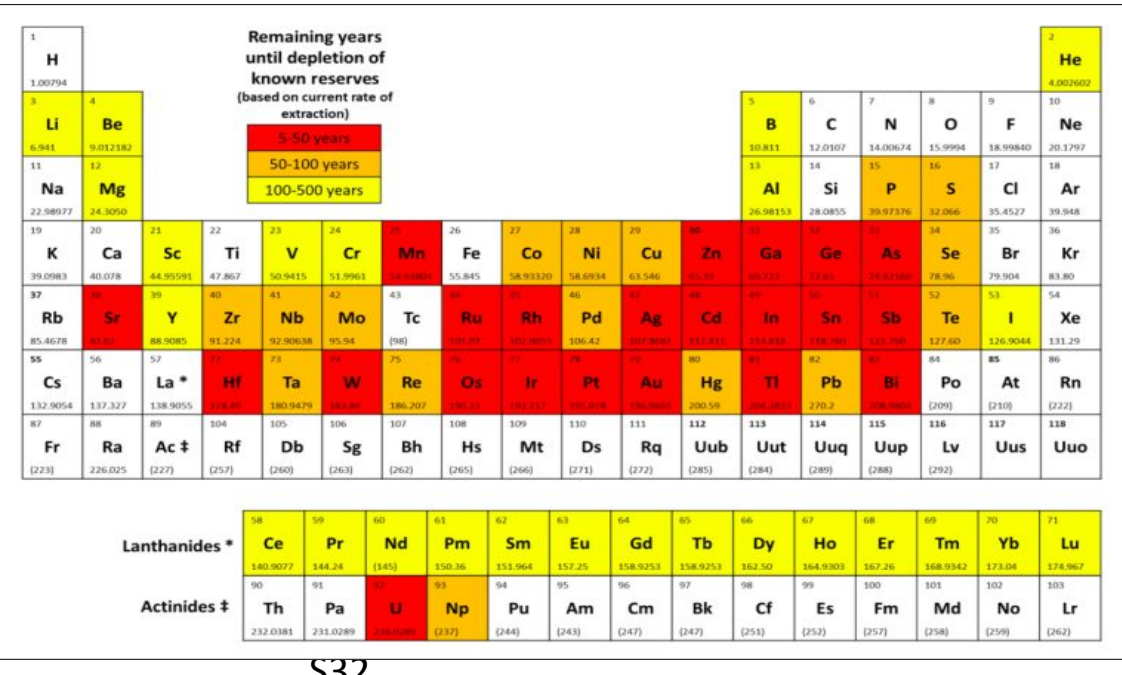


Energy (First

Pass)

\begin{tabular}{|r|r|r|}
\hline Reaction run between 0 to $70^{\circ} \mathrm{C}$ & Green Flag & $\mathrm{X}$ \\
\hline $\begin{array}{r}\text { Reaction run between }-20 \text { to } 0 \text { or } 70 \text { to } \\
140^{\circ} \mathrm{C}\end{array}$ & Amber Flag & \\
\hline Reaction run below -20 or above $140^{\circ} \mathrm{C}$ & Red Flag & \\
\hline
\end{tabular}

\section{Batch/flow}

Flow

Batch

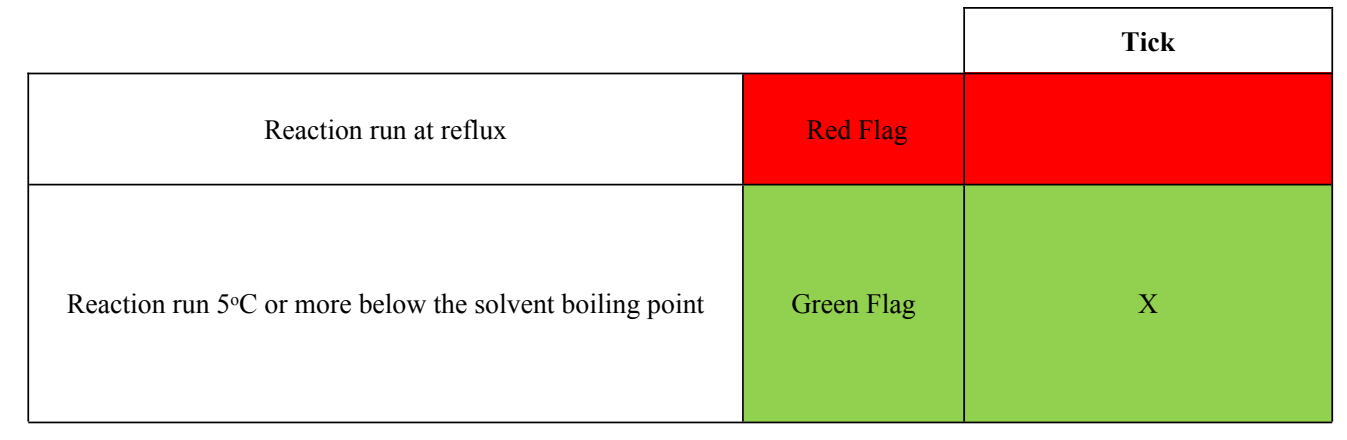

\begin{tabular}{|c|c|c|}
\hline \multicolumn{2}{|l|}{ Work Up } & List \\
\hline $\begin{array}{c}\text { quenching } \\
\text { filtration } \\
\text { centrifugation } \\
\text { crystallisation } \\
\text { Low temperature distillation/evaporation/sublimation }(<140 \\
\left.{ }^{\circ} \mathrm{C} \text { at atmospheric pressure }\right)\end{array}$ & Green Flag & $\begin{array}{l}\text { Filtration, Low temperature } \\
\text { distillation }\end{array}$ \\
\hline solvent exchange, quenching into aqueous solvent & Amber Flag & Extraction \\
\hline $\begin{array}{c}\text { chromatography/ion exchange } \\
\text { high temperature distillation/evaporations/sublimation }(> \\
140^{\circ} \mathrm{C} \text { at atmospheric pressure) } \\
\text { multiple recrystallisation }\end{array}$ & Red Flag & \\
\hline
\end{tabular}




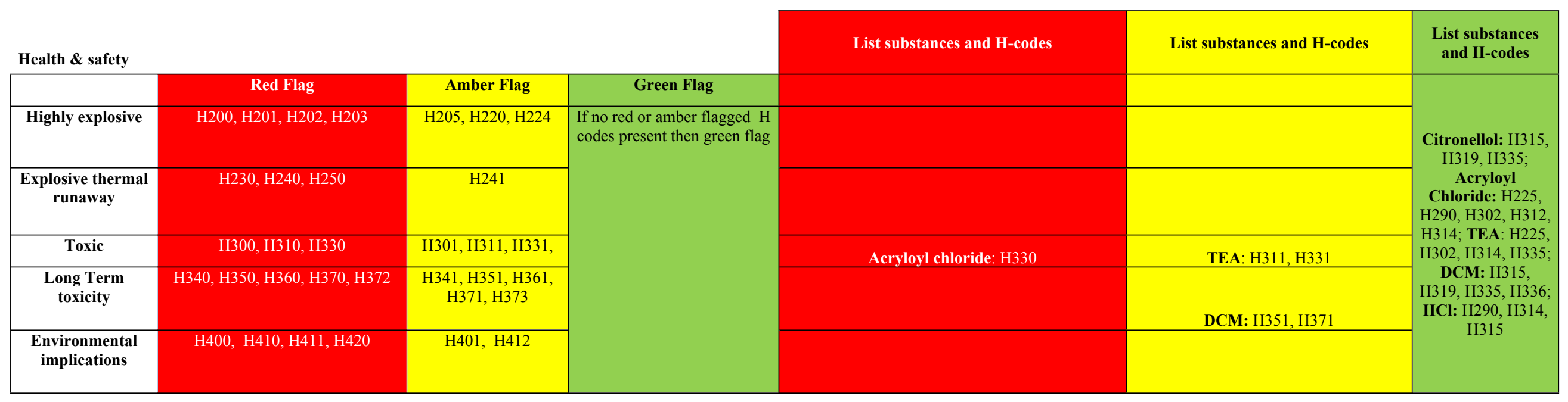

